TRANSACTIONS OF THE

AMERICAN MATHEMATICAL SOCIETY

Volume 351, Number 3, March 1999, Pages 947-983

S 0002-9947(99)02018-8

\title{
DISCRETE THRESHOLD GROWTH DYNAMICS ARE OMNIVOROUS FOR BOX NEIGHBORHOODS
}

\author{
TOM BOHMAN
}

\begin{abstract}
In the discrete threshold model for crystal growth in the plane we begin with some set $A_{0} \subset \mathbf{Z}^{2}$ of seed crystals and observe crystal growth over time by generating a sequence of subsets $A_{0} \subset A_{1} \subset A_{2} \subset \cdots$ of $\mathbf{Z}^{2}$ by a deterministic rule. This rule is as follows: a site crystallizes when a threshold number of crystallized points appear in the site's prescribed neighborhood. The growth dynamics generated by this model are said to be omnivorous if $A_{0}$ finite and $A_{i+1} \neq A_{i} \forall i$ imply $\bigcup_{i=0}^{\infty} A_{i}=\mathbf{Z}^{2}$. In this paper we prove that the dynamics are omnivorous when the neighborhood is a box (i.e. when, for some fixed $\rho$, the neighborhood of $z$ is $\left.\left\{x \in \mathbf{Z}^{2}:\|x-z\|_{\infty} \leq \rho\right\}\right)$. This result has important implications in the study of the first passage time when $A_{0}$ is chosen randomly with a sparse Bernoulli density and in the study of the limiting shape to which $n^{-1} A_{n}$ converges.
\end{abstract}

\section{INTRODUCTION}

Consider the following discrete model for crystal growth in the plane. We think of our crystal as some subset of the two dimensional integer lattice. The model has two parameters: a threshold $\theta$ and a neighborhood $\mathcal{N}$ of the origin $(\mathcal{N}$ is a finite subset of $\mathbf{Z}^{2}$ ). We begin with some set of seed crystals, $A_{0} \subset \mathbf{Z}^{2}$, and the crystal 'grows' according to iterative applications of

$$
A_{i+1}=A_{i} \cup\left\{x \in \mathbf{Z}^{2}:\left|(x+\mathcal{N}) \cap A_{i}\right| \geq \theta\right\} .
$$

In words, a point in the lattice crystallizes as soon as enough of its neighbors (as defined by $\mathcal{N}$ ) are crystallized. The growth dynamics that this model generates are called discrete threshold growth dynamics.

This model was recently introduced by Janko Gravner and David Griffeath [GG1], [GG2]. In their words:

This extremely simple model arose in connection with our previous empirical and theoretical studies of mathematical prototypes for excitable media and crystal growth. In various contexts it captures the essential qualitative and quantitative features of wave propagation.

Gravner and Griffeath have, among other things, been concerned with the following two questions. First, if $A_{0}$ is finite and $A_{i} \neq A_{i+1} \forall i$, then what is the limiting shape of $A_{n} / n$ ? They have given an elegant, interesting answer to this question, but this answer only applies when $A_{\infty}:=\bigcup_{i \geq 0} A_{i}=\mathbf{Z}^{2}$. In rough terms, Gravner and Griffeath's second question is: if $A_{0}$ is chosen at random (each $x \in \mathbf{Z}^{2}$ is in

Received by the editors August 19, 1996 and, in revised form, February 7, 1997.

1991 Mathematics Subject Classification. Primary 60K35; Secondary 05D99.

Key words and phrases. Threshold growth, cellular automata. 
$A_{0}$ with a fixed probability $p$ ), how long does it take for the crystal to occupy a particular site? Gravner and Griffeath have also answered this second question, but their theorem only applies when the dynamics satisfy the following (or a slightly weaker) condition

$$
A_{0} \text { finite and } A_{i} \neq A_{i+1} \forall i \Rightarrow A_{\infty}=\mathbf{Z}^{2} .
$$

Discrete threshold growth dynamics (which are determined by the choice of $\mathcal{N}$ and $\theta)$ are omnivorous if they satisfy (1).

So the work of Gravner and Griffeath begs a combinatorial question which is interesting in its own right: what choices of $\mathcal{N}$ and $\theta$ yield dynamics that satisfy (1)? Discrete threshold growth dynamics are not always omnivorous. For example, if both $A_{0}$ and $\mathcal{N}$ contain only elements from the even sublattice of $\mathbf{Z}^{2}$, then $A_{\infty}$ is contained in the even sublattice. Is there a statement similar to (1) that is true in general? Restricting our attention to symmetric neighborhoods (i.e. $\mathcal{N}=-\mathcal{N}$ ) it seems that the most aggressive conjecture that can be made here is:

Conjecture. If $\mathcal{N}=-\mathcal{N}$ and $A_{0}$ is finite, then $A_{\infty}$ is the union of a finite number of cosets of subgroups of $\mathbf{Z}^{2}$.

In their papers Gravner and Griffeath are primarily concerned with box neighborhoods: $\mathcal{N}=\left\{z \in \mathbf{Z}^{2}:\|z\|_{\infty} \leq \rho\right\}$ for some constant $\rho$. It appears that this choice is made both because box neighborhoods are a natural first case to consider and because the way that box neighborhoods mesh with the underlying lattice simplifies computations. In [GG2] they conjectured that if $\mathcal{N}$ is a box neighborhood, then the dynamics are omnivorous. Here we settle that conjecture.

Theorem 1. Discrete threshold growth dynamics are omnivorous for box neighborhoods.

We now give an introduction to the proof of Theorem 1. Henceforth we consider only those choices of $\theta, \mathcal{N}$ and finite $A_{0}$ for which the crystal does not stop growing. We begin with a general result - a lemma that applies for any choice of symmetric neighborhood. We prove this lemma by applying a simple weight function argument (for some other examples of how weight function arguments are used in combinatorics see [C, pp. 715-717], [ES]). A point in the lattice will be considered 'heavy' or 'of high energy' if it has not been crystallized but has many neighbors which are crystallized. To be precise, at time $t$ the weight of a point $x \in \mathbf{Z}^{2}$ is defined to be

$$
w_{t}(x)= \begin{cases}\left|(x+\mathcal{N}) \cap A_{t}\right|, & x \notin A_{t}, \\ 0, & x \in A_{t} .\end{cases}
$$

For an arbitrary subset $X \subset \mathbf{Z}^{2}$ we define the total weight over $\mathrm{X}$ at time $t$ as

$$
W_{t}=W_{t}(X)=\sum_{x \in X} w_{t}(x) .
$$

Lemma 2. If $\mathcal{N}=-\mathcal{N}$, then for $n$ sufficiently large the average of $\left|(x+\mathcal{N}) \cap A_{n}\right|$ over the elements of $A_{n} \backslash A_{0}$ is greater than $2 \theta-1$.

Proof. Let $c=\left|A_{0}\right||\mathcal{N}|$ and choose $n$ so that $\left|A_{n} \backslash A_{0}\right|>c$. We use the above weight function with $X=A_{n}$. Clearly $W_{0} \leq c$ and $W_{n}=0$. Now consider the change in the total weight that occurs as each element of $A_{n} \backslash A_{0}$ crystallizes. If $x \in A_{k+1} \backslash A_{k}$, then as $x$ crystallizes the total weight will go down by $\left|(x+\mathcal{N}) \cap A_{k}\right|$ 
as the weight of $x$ will go to zero. But, the total weight will simultaneously increase by $\left|(x+\mathcal{N}) \cap\left(A_{n} \backslash A_{k+1}\right)\right|$ since the weight of anything in the neighborhood of $x$ which isn't already crystallized (or simultaneously being crystallized) goes up by one. So, we can express the change in the total weight that is caused by the crystallization of $x$ as $\Delta_{x}=-\left|(x+\mathcal{N}) \cap A_{k}\right|+\left|(x+\mathcal{N}) \cap\left(A_{n} \backslash A_{k+1}\right)\right|$. The total change in the total weight is given by the sum of the changes over all $x$ :

$$
W_{n}-W_{0}=\sum_{x \in A_{n} \backslash A_{0}} \Delta_{x} .
$$

We've already determined that the left hand side of this equation must be at least $-c$. Since there are more than $c$ terms in the summation, the average of $\Delta_{x}$ over $A_{n} \backslash A_{0}$ is greater than -1 . Since, for $x \in A_{k+1} \backslash A_{k}$,

$$
\begin{aligned}
\left|(x+\mathcal{N}) \cap A_{n}\right| & \geq\left|(x+\mathcal{N}) \cap\left(A_{n} \backslash A_{k+1}\right)\right|+\left|(x+\mathcal{N}) \cap A_{k}\right| \\
& =2\left|(x+\mathcal{N}) \cap A_{k}\right|+\Delta_{x} \\
& \geq 2 \theta+\Delta_{x}
\end{aligned}
$$

we can conclude that the average of $\left|(x+\mathcal{N}) \cap A_{n}\right|$ over $A_{n} \backslash A_{0}$ is greater than $2 \theta-1$.

We now restrict our attention to box neighborhoods and give a brief overview of how Lemma 2 is used to prove Theorem 1. We divide the proof into cases based on the value of $\theta$. First, note that for $\theta>(2 \rho+1) \rho$ the final crystal, $A_{\infty}$, is contained in any rectangle that contains $A_{0}$. Thus we can restrict our attention to $0<\theta \leq \rho(2 \rho+1)$. In the case of box neighborhoods with $\theta \leq \rho^{2}$ we conclude from Lemma 2 that there exists a point $x$ and a time $n$ for which $\left|(x+\mathcal{N}) \cap A_{n}\right| \geq 2 \theta$. This local event will be enough to 'generate' the entire plane.

On the other hand, when $\rho^{2}<\theta \leq \rho(2 \rho+1)$ we employ a much more sophisticated indirect approach which uses the full power of Lemma 2. We begin this indirect proof by assuming that $A_{\infty} \neq \mathbf{Z}^{2}$. We then dub certain connected subsets of $A_{\infty}$ 'jagged blocks' (the precise definition is given below). Loosely speaking, the jagged blocks are the parts of $A_{\infty}$ that are particularly dense. The assumption $A_{\infty} \neq \mathbf{Z}^{2}$ implies that jagged blocks are bounded in size. It also happens that jagged blocks only occur near the seed crystals. With jagged blocks more or less excluded one can show that the average of $\left|(x+\mathcal{N}) \cap A_{\infty}\right|$ over an appropriate set is small. This contradicts a modified version of Lemma 2 .

The rest of this paper is organized as follows. We end this section with a discussion of how the results in this paper might be extended to other neighborhoods. Section 2 contains the proof for the case $\theta \leq \rho^{2}$, and section 3 consists of the proof for $\rho^{2}<\theta \leq 2 \rho^{2}+\rho$ up to two technical details. These technical details are handled in sections 4 and 5 .

Can the methods developed in this paper be used to show that discrete threshold growth dynamics are omnivorous for neighborhoods other than box neighborhoods? While Lemma 2 holds in considerable generality, many of the arguments in the rest of the paper are geometric counting arguments that use the box neighborhood assumption heavily. These counting arguements cannot be easily modified to work for a more general class of neighborhoods (i.e. a large set of 'nice' neighborhoods that perhaps contains the box neighborhoods. One candidate for a more general class of neighborhoods is the obese neighborhoods defined in [GG1]). Such a general theorem would require some substantial new ideas in order to either verify that these 
counting arguments work for the more general class of neighborhood or eliminate the need for these counting arguments in the proof. As the difficulty of the proof presented here increases with threshold, one would expect that the difficulty of such a general theorem would also increase with the threshold.

On the other hand, one can apply these methods to other specific neighborhoods by modifying the geometric counting arguments accordingly. For example, the arguments given in section 2 can easily be modified to show that the dynamics are omnivorous for neighborhoods of the form $\mathcal{N}=\left\{z \in \mathbf{Z}^{2}:\|z\|_{1} \leq \rho\right\}$ (these are diamond neighborhoods) with $\theta \leq \rho^{2} / 2-\rho / 2$. This modification could certainly be repeated for other neighborhoods (and perhaps could - with a lot more work - be extended to larger thresholds in the diamond neighborhood case). This flexibility in the method suggests that discrete threshold growth dynamics are in fact omnivorous for some general class of 'nice' neighborhoods.

$$
\text { 2. } \theta \leq \rho^{2}
$$

In this case, if $A_{i}$ contains a full $\rho \times \rho$ square, then $A_{\infty}=\mathbf{Z}^{2}$. To see this, suppose $A_{i}$ contains a $\rho \times \rho$ square $S$. The neighborhood of any element of

$$
S_{1}:=\left\{z \in \mathbf{Z}^{2}: \exists s \in S \text { such that }\|z-s\|_{\infty}=1\right\}
$$

contains $\rho^{2}$ elements of $S$, and therefore $S_{1} \subset A_{i+1}$. By induction, then,

$$
S_{k}:=\left\{z \in \mathbf{Z}^{2}: \exists s \in S \text { such that }\|z-s\|_{\infty} \leq k\right\} \subset A_{i+k} .
$$

This implies $A_{\infty}=\mathbf{Z}^{2}$.

Lemma 2 implies the existence of a point $x$ and a time $n$ for which $\left|(x+\mathcal{N}) \cap A_{n}\right| \geq$ $2 \theta$. We will show that a central $\rho \times \rho$ sub-square of this neighborhood eventually fills in (technically, the argument we give shows that the central $\rho \times \rho$ sub-square fills in when $\rho$ is odd and that the central $(\rho+1) \times(\rho+1)$ sub-square fills in when $\rho$ is even). According to the preceding paragraph, this is enough to prove Theorem 1 when $\theta \leq \rho^{2}$. For ease of notation we may begin by assuming that the neighborhood of the origin contains at least $2 \theta$ elements of $A_{0}$.

Lemma 3. Suppose $\left|A_{0} \cap \mathcal{N}\right| \geq 2 \theta$. If $|x|+|y| \leq \rho$, then either $(x, y) \in A_{|x|+|y|+1}$ or $(-x,-y) \in A_{|x|+|y|+1}$.

Proof. We proceed by induction on $|x|+|y|$. Clearly, the origin is contained in $A_{1}$. Suppose the assertion holds for all points $(w, z)$ for which $|w|+|z|<|x|+|y|$, and assume without loss of generality that $x \geq y \geq 0$. Let $\mathcal{N}^{\prime}=(x, y)+\mathcal{N}$, and $\mathcal{N}^{\prime \prime}=(-x,-y)+\mathcal{N}$. Consider the parts of $\mathcal{N}$ that are covered by $\mathcal{N}^{\prime}$ and $\mathcal{N}^{\prime \prime}$ (for example, if either $x$ or $y$ is zero, then $\mathcal{N}^{\prime} \cup \mathcal{N}^{\prime \prime}=\mathcal{N}$. Since $\left|\mathcal{N} \cap A_{0}\right| \geq 2 \theta$ either $\mathcal{N}^{\prime}$ or $\mathcal{N}^{\prime \prime}$ must contain $\theta$ elements of $A_{0}$, and the result follows $)$. In general, $\mathcal{N} \backslash\left(\mathcal{N}^{\prime} \cup \mathcal{N}^{\prime \prime}\right)$ consists of two $x \times y$ rectangular regions in opposite corners of $\mathcal{N}$. Furthermore, $\mathcal{N}^{\prime} \cap \mathcal{N}^{\prime \prime}$ contains the rectangle with corners $(y, x),(-y, x),(-y,-x)$, and $(y,-x)$. Call this rectangle minus its corner points $R$. By the inductive assumption

$$
a:=\left|R \cap A_{|x|+|y|}\right|>1 / 2[(2 x+1)(2 y+1)-4]=2 x y+x+y-3 / 2 .
$$

Trivially, $b:=\left|\left(\mathcal{N} \backslash \mathcal{N}^{\prime} \backslash \mathcal{N}^{\prime \prime}\right) \cap A_{|x|+|y|}\right| \leq 2 x y$. The set $\mathcal{N}^{\prime} \cup \mathcal{N}^{\prime \prime}$ covers the rest of $\mathcal{N}$ (i.e. $\left.\mathcal{N} \backslash R \backslash\left(\mathcal{N} \backslash \mathcal{N}^{\prime} \backslash \mathcal{N}^{\prime \prime}\right)\right)$. There are at least $2 \theta-a-b$ elements of $A_{|x|+|y|}$ in this region. So, either $\mathcal{N}^{\prime}$ or $\mathcal{N}^{\prime \prime}$ has an intersection with $A_{|x|+|y|}$ of cardinality at 
least

$$
\begin{aligned}
1 / 2(2 \theta-a-b)+a & =\theta+a / 2-b / 2 \\
& \geq \theta+x y+x / 2+y / 2-3 / 4-x y \\
& =\theta+x / 2+y / 2-3 / 4 . \quad \square
\end{aligned}
$$

Note that this is already enough to prove Theorem 1 when $\theta \leq \rho^{2} / 2$. This is due to the fact that we now have at least a $\rho \times \rho$ square that is half filled in. When $\rho$ is odd this is the square bounded by $( \pm(\rho-1) / 2, \pm(\rho-1) / 2)$, and when $\rho$ is even this is the $(\rho+1) \times(\rho+1)$ square bounded by $( \pm \rho / 2, \pm \rho / 2)$. This half-full square will be completely filled with crystal in one iteration of the growth rule. This happens because every element of the square can 'see' every other element and the number of crystals already in the square exceeds $\theta$.

A slightly more complicated calculation is needed to complete the proof when $\rho^{2} / 2<\theta \leq \rho^{2}$. We now assume for ease of notation that the neighborhood of the origin contains at least $2 \theta$ elements of $A_{0}$ and that for ordered pairs $(x, y)$ satisfying $|x|+|y| \leq \rho$ either $(x, y) \in A_{0}$ or $(-x,-y) \in A_{0}$. We will show by induction on $|x|+|y|$ that if $|x|,|y| \leq\lfloor\rho / 2\rfloor$, then $(x, y) \in A_{|x|+|y|+1}$. This will complete the proof because $A_{\rho+1}$ will contain a $\rho \times \rho$ square. We achieve $|x|,|y| \leq\lfloor\rho / 2\rfloor \Rightarrow$ $(x, y) \in A_{|x|+|y|+1}$ in two phases, always assuming without loss of generality that $x \geq 0$ and $y \geq 0$.

Phase 1. $|x|+|y| \leq\lfloor\rho / 2\rfloor$.

There are $2 \rho^{2}+2 \rho+1$ points $(z, w)$ which satisfy $|z|+|w| \leq \rho$. By our assumptions on $A_{0}$ at least half of these are crystallized. Of these, the point $(x, y)$ can see all but $x^{2}+y^{2}$. On the other hand the inductive hypothesis implies that all points of the form $(z, w)$ where $|z|+|w|<|x|+|y|$ are in $A_{|x|+|y|}$. The point $(x, y)$ can see all of these. So,

$$
\begin{aligned}
((x, y)+\mathcal{N}) \cap A_{|x|+|y|} \geq & 1 / 2\left(2 \rho^{2}+2 \rho+1\right)-x^{2}-y^{2} \\
& +1 / 2\left[2(x+y-1)^{2}+2(x+y-1)+1\right] \\
= & \rho^{2}+\rho+2 x y-x-y+1 \\
> & \rho^{2}+2 x y \geq \theta .
\end{aligned}
$$

Phase 2. $|x|+|y|>\lfloor\rho / 2\rfloor$.

Here we make nearly the same calculation. The difference is that we cannot assume that all points $(z, w)$ where $|z|+|w|<|x|+|y|$ are in $A_{|x|+|y|}$; only those $(z, w)$ for which $|z|,|w| \leq\lfloor\rho / 2\rfloor$ are known to be in $A_{|x|+|y|}$. There are $4(x+y-1-\lfloor\rho / 2\rfloor)^{2}$ points $(z, w)$ that satisfy $|z|+|w|<|x|+|y|$ yet fail to satisfy $|z|,|w| \leq\lfloor\rho / 2\rfloor$. One half of this quantity must be subtracted from the bound we achieved in Phase 1.

$$
\begin{aligned}
((x, y)+\mathcal{N}) \cap A_{|x|+|y|} & >\rho^{2}+2 x y-2(x+y-1-\lfloor\rho / 2\rfloor)^{2} \\
& \geq \rho^{2}+2 x y-2(x+y-\lfloor\rho / 2\rfloor)^{2} .
\end{aligned}
$$

If $u=x+y$ is fixed this quantity is minimized when $x=\lfloor\rho / 2\rfloor$ and $y=u-\lfloor\rho / 2\rfloor$, so

$$
\begin{aligned}
((x, y)+\mathcal{N}) \cap A_{|x|+|y|} & >\rho^{2}+2\lfloor\rho / 2\rfloor(u-\lfloor\rho / 2\rfloor)-2(u-\lfloor\rho / 2\rfloor)^{2} \\
& =\rho^{2}+2(u-\lfloor\rho / 2\rfloor)(2\lfloor\rho / 2\rfloor-u) \\
& >\theta .
\end{aligned}
$$


In this case the proof will be indirect. We will begin by assuming that $A_{\infty} \neq \mathbf{Z}^{2}$. This will imply that certain structures do not appear in $A_{\infty}$. The absence of these structures will imply that the average of $\left|(x+\mathcal{N}) \cap A_{\infty}\right|$ over an appropriate set is less than $2 \theta+1 / 2$. This contradicts a modified version of Lemma 2. Before discussing the proof in more detail we must describe the 'certain structures' and the 'appropriate set'.

We are interested in parts of $\mathbf{Z}^{2}$ where $A_{\infty}$ is particularly dense. For any $x \in \mathbf{Z}^{2}$ let $S_{x}$ be the $\rho \times \rho$ square whose lower left-hand corner is $x$ :

$$
S_{x}:=\left\{x+\alpha e_{1}+\beta e_{2}: 0 \leq \alpha, \beta<\rho\right\}
$$

(throughout this paper $e_{1}=(1,0)$ and $\left.e_{2}=(0,1)\right)$. Let $V$ be the collection of all $\rho \times \rho$ squares contained in $A_{\infty}$ (i.e. $V=\left\{S_{x}: S_{x} \subset A_{\infty}\right\}$ ). We form a graph $G$ on vertex set $V$ by connecting two squares if the cardinality of their intersection is exactly $(\rho-1)(\rho)$; that is, $S_{x}$ is adjacent to $S_{y}$ if $x=y \pm e_{1}$ or $x=y \pm e_{2}$. For any connected component $C$ of $G$ the subset $\bigcup_{S_{x} \in C} S_{x}$ of $A_{\infty}$ will be called a jagged block. We will think of these jagged blocks as the places where $A_{\infty}$ is very dense. It turns out that when $A_{\infty} \neq \mathbf{Z}^{2}$ jagged blocks are bounded in size. It also happens that jagged blocks always occur near the seed crystals. Since $A_{0}$ is a finite set, this implies that $A_{\infty}$ contains only finitely many jagged blocks. Since, as we will see below, infinitely many or infinitely large jagged blocks are needed for the average of $\left|(x+\mathcal{N}) \cap A_{n}\right|$ to be large (i.e. greater than $2 \theta+1 / 2$ ), these facts concerning jagged blocks yield a contradiction.

We now discuss some of the elementary local properties of an arbitrary jagged block $\mathcal{B}$. These properties follow from the fact that $A_{\infty}$ is stable under applications of the crystal growth rule (i.e. for $x \in \mathbf{Z}^{2} \backslash A_{\infty}$ we have $\left|(x+\mathcal{N}) \cap A_{\infty}\right|<\theta$ ). For $S_{x}, S_{y} \subset \mathcal{B}$ let $P_{x, y}$ be a shortest path from $S_{x}$ to $S_{y}$ in $G$. We begin by noting that $P_{x, y}$ is always as direct as possible; that is, the number $\left|P_{x, y}\right|$ of edges in $P_{x, y}$ satisfies

$$
\left|P_{x, y}\right|=\|x-y\|_{1} .
$$

Proof. Let $P_{x, y}=S_{p_{1}}, S_{p_{2}}, \ldots, S_{p_{l}}$. For $1 \leq j \leq l-1$ let $m_{j}=p_{j+1}-p_{j}$. We prove (4) by showing that at most one element of the set $\left\{ \pm e_{1}\right\}$ appears in $m_{1}, m_{2}, \ldots, m_{l-1}$ (and that at most one element of the set $\left\{ \pm e_{2}\right\}$ appears in $\left.m_{1}, \ldots, m_{l-1}\right)$. Throughout this paper we will refer to the elements of $\left\{ \pm e_{1}, \pm e_{2}\right\}$ that appear in $m_{1}, m_{2}, \ldots, m_{l}$ as the basis vectors of $P_{x, y}$.

Assume for the sake of contradiction that $e_{1}$ and $-e_{1}$ appear in $m_{1}, \ldots, m_{l-1}$. This implies that there exists $\alpha<\beta$ such that $m_{\alpha} \in\left\{ \pm e_{1}\right\}, m_{\beta}=\left\{ \pm e_{1}\right\} \backslash m_{\alpha}$, and $m_{\gamma} \in\left\{ \pm e_{2}\right\}$ for $\alpha<\gamma<\beta$. Assume without loss of generality that $m_{\alpha}=e_{1}$, $m_{\beta}=-e_{1}$ and $p_{\beta+1}=p_{\alpha}+(\beta-\alpha-1) e_{2}$. In this setting we begin by establishing the following:

$$
\exists q \in\left\{p_{\alpha}+i e_{2}: 1 \leq i \leq \beta-\alpha-1\right\} \text { such that } S_{q} \not \subset \mathcal{B} .
$$

This holds because $P_{x, y}$ is a shortest path (i.e. if (5) does not hold, then there exists a path from $x$ to $y$ shorter than $\left.P_{x, y}\right)$. One consequence of $(5)$ is that $\beta-\alpha-1>\rho$. The more important consequence of (5) is that there exists $h \in$ $\left\{\mathbf{Z}^{2} \backslash A_{\infty}\right\} \cap\left\{p_{\alpha}+i e_{2}: \rho \leq i \leq \beta-\alpha-2\right\}$. Letting $A=\bigcup_{i=\alpha}^{\beta} S_{p_{i}}$ we have $\left|(h+\mathcal{N}) \cap A_{\infty}\right| \geq|(h+\mathcal{N}) \cap A| \geq 2 \rho^{2}+\rho \geq \theta$, which is a contradiction. 
We now give a list of Corollaries of (4) (these are given in equations (6)-(12)). These facts will be very useful in our global characterization of jagged blocks - our discussion of how jagged blocks can be 'built' by the crystal - and are somewhat technical. However, some of them (namely (8), (10) and (11)) are fairly useful in developing an intuitive understanding of what a jagged block looks like.

The first four Corollaries concern 'internal' properties of a jagged block. Consider the following setting: $\mathcal{B}$ is a jagged block, $S_{x}, S_{y} \subset \mathcal{B}$, and there exists $e_{x, y} \in\left\{e_{1}, e_{2}\right\}$ such that

$$
\left(\left(S_{x}+\mathbf{Z} e_{x, y}\right) \cup\left(S_{x}+\rho e_{x, y}^{\prime}+\mathbf{Z} e_{x, y}\right) \cup\left(S_{x}-\rho e_{x, y}^{\prime}+\mathbf{Z} e_{x, y}\right)\right) \cap S_{y} \neq \emptyset
$$

where $e_{x, y}^{\prime}=\left\{e_{1}, e_{2}\right\} \backslash e_{x, y}$. Two such squares will be called snug. Let $R_{x, y}$ be the smallest rectangle in $\mathbf{Z}^{2}$ (set of the form $\left\{(x, y): x^{\prime}<x<x^{\prime \prime}\right.$ and $\left.y^{\prime}<y<y^{\prime \prime}\right\}$ ) containing $S_{x} \cup S_{y}$. It follows from (4) that

$$
z \in\left(S_{x}+\mathbf{Z} e_{x, y}\right) \cap R_{x, y} \backslash \mathcal{B} \Rightarrow(z+\mathcal{N}) \cap\left(S_{y}+\mathbf{Z} e_{x, y}\right) \subset \mathcal{B} .
$$

Proof. Let $x=\left(x_{1}, x_{2}\right)$ and $y=\left(y_{1}, y_{2}\right)$. Assume without loss of generality that $x_{1} \leq y_{1}, x_{2} \leq y_{2}$ and $e_{x, y}=e_{2}$. Let $z$ be an arbitrary element of $\left(S_{x}+\mathbf{Z} e_{2}\right) \cap R_{x, y} \backslash \mathcal{B}$. Note that we must have $z_{2} \geq x_{2}+\rho$.

Let $S_{u:=\left(u_{1}, u_{2}\right)}$ be the square in $P_{x, y}$ for which $u_{2}=z_{2}-\rho$ and $S_{u}+e_{2} \in P_{x, y}$. By (4) $x_{1} \leq u_{1} \leq y_{1}$ and $x_{2} \leq u_{2} \leq y_{2}$. But, since we cannot have $z \in S_{u}+e_{2}$, we must also have $u_{1}>z_{1}$.

Now, let $v:=\left(v_{1}, v_{2}\right)$ be an arbitrary element of $(z+\mathcal{N}) \cap\left(S_{y}+\mathbf{Z} e_{2}\right)$. We may assume $v \notin S_{y}$ (i.e. $\left.v_{2}<y_{2}\right)$. Let $S_{w:=\left(w_{1}, w_{2}\right)}$ be the square in $P_{x, y}$ for which $w_{2}=v_{2}$ and $S_{w}+e_{2} \in P_{x, y}$. By (4), $w_{1} \leq y_{1}$ and hence $w_{1} \leq v_{1}$. Since $S_{w}$ follows $S_{u}$ in $P_{x, y}$ we have $w_{1} \geq u_{1}>z_{1}$ which implies $w_{1}+\rho>z_{1}+\rho \geq v_{1}$. Since $w_{1} \leq v_{1}<w_{1}+\rho, v \in S_{w}$.

Equation (6) has a number of immediate Corollaries in the following, slightly more restrictive situation. Consider squares $S_{x}, S_{y} \subset \mathcal{B}$ for which there exists $e_{x, y} \in\left\{e_{1}, e_{2}\right\}$ such that $\left(S_{x}+\mathbf{Z} e_{x, y}\right) \cap S_{y} \neq \emptyset$ (equivalently $\left(S_{y}+\mathbf{Z} e_{x, y}\right) \cap S_{x} \neq \emptyset$ ). We will say that two such squares are tight. Note that tight squares are also snug. In order to state the Corollaries of (6) precisely we must first 'dissect' $R_{x, y}$ (again, this is the smallest rectangle containing $S_{x} \cup S_{y}$ ). Let

$$
R_{x, y}^{c}=\left(R_{x, y} \cap\left(S_{x}+e_{x, y} \mathbf{Z}\right) \cap\left(S_{y}+e_{x, y} \mathbf{Z}\right)\right) \backslash S_{x} \backslash S_{y}
$$

and for $u \in\{x, y\}$ let

$$
R_{x, y}^{u}=\left(\left(S_{u}+\mathbf{Z}^{2} e_{x, y}\right) \cap R_{x, y}\right) \backslash S_{x} \backslash S_{y} \backslash R_{x, y}^{c} .
$$

Note that $R_{x, y}=R_{x, y}^{c} \cup R_{x, y}^{x} \cup R_{x, y}^{y} \cup S_{x} \cup S_{y}$ and that there exists a vector $d_{x, y} \in\left\{ \pm \rho e_{1} \pm \rho e_{2}\right\}$ such that $R_{x, y}^{x}+d_{x, y}=R_{x, y}^{y}$. The first immediate Corollary of (6) concerns what happens 'between' tight squares $S_{x}$ and $S_{y}$ :

$$
R_{x, y}^{c} \subset \mathcal{B} .
$$

From (7) it follows immediately that

$$
\mathcal{B} \text { is horizontally and vertically convex. }
$$

To be precise, if $x, x+n e_{1} \in \mathcal{B}$ for some $n \in \mathbf{N}$, then $x+e_{1}, x+2 e_{1}, \ldots, x+(n-1) e_{1} \in$ $\mathcal{B}$, and if $x, x+n e_{2} \in \mathcal{B}$ for some $n \in \mathbf{N}$, then $x+e_{2}, x+2 e_{2}, \ldots, x+(n-1) e_{2} \in \mathcal{B}$. Now, for $z \in R_{x, y}^{x}$ let $z^{\prime}=z+d_{x, y}$. It also follows immediately from (6) that

$$
\forall z \in R_{x, y}^{x} \text { either } z \in \mathcal{B} \text { or } z^{\prime} \in \mathcal{B} \text {. }
$$


What happens at the 'boundary' of a jagged block? Let $\mathcal{B}$ be a jagged block defined by a component $C$ of $G$. Let $C^{\prime}=\left\{S_{x} \notin C: \exists S_{y} \in C\right.$ such that $\left|S_{y} \cap S_{x}\right|=$ $\rho(\rho-1)\}$. It follows immediately from the definitions that for every $S_{x} \in C^{\prime}$ we have $S_{x} \backslash \mathcal{B} \backslash A_{\infty} \neq \emptyset$; the 'boundary' of $\mathcal{B}$ is defined by elements of $\mathbf{Z}^{2} \backslash A_{\infty}$. We will call the elements of $\left(\bigcup_{S_{x} \in C^{\prime}} S_{x}\right) \backslash B \backslash A_{\infty}$ pegs. In other words, pegs are elements of $\mathbf{Z}^{2} \backslash A_{\infty}$ that are adjacent to $\mathcal{B}$. We further define $z \in \mathbf{Z}^{2} \backslash A_{\infty}$ for which $\left|\left\{z \pm e_{1}, z \pm e_{2}\right\} \cap \mathcal{B}\right|=2$ to be corner pegs.

Suppose $z$ is a corner peg with $a, b \in \mathcal{B}$ and $\|z-a\|_{1},\|z-b\|_{1}=1$. By (8) we must have $\{a, b\}=\left\{z+\alpha e_{1}, z+\beta e_{2}\right\}$ for some $\alpha, \beta \in\{1,-1\}$. Let $a \in S_{x} \subset \mathcal{B}$ and $b \in S_{y} \subset \mathcal{B}$. Applying (4) to $P_{x, y}$ gives

$$
S_{\mathcal{B}, z}:=\left\{z+i \alpha e_{1}+j \beta e_{2}: 1 \leq i, j \leq \rho\right\} \subset \mathcal{B} .
$$

Now suppose there exists $y \in \mathcal{B} \cap\left(z-\alpha e_{1} \mathbf{N}-\beta e_{2} \mathbf{N}\right)$. Let $S_{w} \subset \mathcal{B}$ be a square containing $y$. Now consider the path in $G$ from $S_{\mathcal{B}, z}$ to $S_{w}$. In particular, consider the first $\rho+1$ squares in this path. By (4) these squares are either $\left\{S_{\mathcal{B}, z}-\alpha i e_{1}\right.$ : $i=0,1, \ldots, \rho+1\}$ or $\left\{S_{\mathcal{B}, z}-\beta i e_{2}: i=0,1, \ldots, \rho+1\right\}$. If $A$ is the union of these $\rho+2$ squares then, in either case, $A \subset z+\mathcal{N}$ and $\left|(z+\mathcal{N}) \cap A_{\infty}\right| \geq \rho(2 \rho+1) \geq \theta$, which is a contradiction. We have shown

$$
\left(p-\alpha e_{1} \mathbf{N}-\beta e_{2} \mathbf{N}\right) \cap \mathcal{B}=\emptyset .
$$

Our final corollary of (4) concerns the basis vectors of an arbitrary $P_{x, y}$. If $u \neq v$ are the basis vectors of $P_{x, y}$, then

$$
S_{x}+u \not \subset \mathcal{B} \Rightarrow \exists p \in S_{x}+u \text { such that } p \notin A_{\infty} \text { and } p+v \in \mathcal{B} .
$$

Proof. Since $S_{x}+u \not \subset \mathcal{B}, m_{1}=v$. Let $k=\min \left\{i: S_{x}+i v+u \subset \mathcal{B}\right\}$. Since there exists a path from $S_{x}$ to $S_{y}$ in $G$, such a $k$ must exist. Let $A=\left(\bigcup_{i=0}^{k} S_{x}+i v\right) \cup$ $\left(S_{x}+k v+u\right)$. By the minimality of $k, p:=\left(S_{x}+(k-1) v+u\right) \backslash A \notin A_{\infty}$. So $p$ is a corner peg. If $p \notin S_{x}+u$, then $\left|(p+\mathcal{N}) \cap A_{\infty}\right| \geq \rho(2 \rho+1) \geq \theta$. This is a contradiction. So, $p \in S_{x}+u$.

With these local facts in hand we now pass to a discussion of the global properties of jagged blocks. We define the diameter of an arbitrary $X \subset \mathbf{Z}^{2}$ to be $d(X):=$ $\max \left\{\|x-y\|_{\infty}: x, y \in X\right\}$.

Lemma 4. If $A_{\infty}$ contains a jagged block $\mathcal{B}$ with $d(\mathcal{B})>2 \rho^{3}$, then $A_{\infty}=\mathbf{Z}^{2}$.

Proof. Suppose $A_{\infty}$ contains a jagged block $\mathcal{B}$ with $d(\mathcal{B})>2 \rho^{3}$. Let $C$ be the component of $G$ that defines $\mathcal{B}$. There exists a time $k$ and a set $C^{\prime} \subset C$ such that $\mathcal{B}^{\prime}:=\bigcup_{S_{x} \in C^{\prime}} S_{x} \subset A_{k}, d\left(\mathcal{B}^{\prime}\right)>2 \rho^{3}$ and $C^{\prime}$ satisfies (4) (if $\mathcal{B}$ is finite let $C^{\prime}=C$ and if $\mathcal{B}$ is infinite let $C^{\prime}$ be the intersection of $C$ with some very large rectangle). We will show that $\mathcal{B}^{\prime}$ alone generates the entire plane. Since the crystal that is generated by $\mathcal{B}^{\prime}$ is contained in the crystal that is generated by $A_{k}$, this implies that $A_{\infty}=\mathbf{Z}^{2}$.

For ease of notation we suppose $A_{0}=\mathcal{B}^{\prime}$; we suppose $A_{0}=\bigcup_{S_{x} \subset C^{\prime}} S_{x}$ for some $C^{\prime} \subset V$ which yields $d\left(A_{0}\right)>2 \rho^{3}$ and for which the subgraph of $G$ that is induced by $C^{\prime}$ is connected and satisfies (4).

Let $l=\min \left\{x:(x, y) \in A_{0}\right\}$ and $r=\max \left\{x:(x, y) \in A_{0}\right\}$. Since $d\left(A_{0}\right)>2 \rho^{3}$ we may assume without loss of generality that $r-l>2 \rho^{3}$. We will show that $A_{\infty}$ contains the set $U:=\left\{(x, y) \notin A_{0}: \exists y^{\prime}<y\right.$ such that $\left.\left(x, y^{\prime}\right) \in A_{0}\right\}$. It follows that $A_{\infty}$ contains $U^{\prime}:=\left\{(x, y): \exists y^{\prime}\right.$ such that $\left.\left(x, y^{\prime}\right) \in A_{0}\right\}$. Now consider an arbitrary 
$(u, v) \in \mathbf{Z}^{2}$. Let $U^{\prime \prime}=\left\{(x, y): v-\rho^{3} \leq y \leq v+\rho^{3}\right\}$. Since $U^{\prime} \subset A_{\infty}$ there exists $j$ such that $U^{\prime} \cap U^{\prime \prime} \subset A_{j}$. It then follows (applying the argument that we use to show that $A_{0}$ generates $U$ to $\left.U^{\prime} \cap U^{\prime \prime}\right)$ that $U^{\prime \prime} \subset A_{\infty}$ and $(u, v) \in A_{\infty}$, and we are done. So it remains to show $U \subset A_{\infty}$.

Throughout this argument we are ignoring the influence of crystallization that occurs outside $U$; we consider the sequence

$$
A_{0}=B_{0} \subset B_{1} \subset B_{2} \subset \cdots \subset U
$$

where

$$
B_{k+1}=B_{k} \cup\left\{x \in U:\left|(x+\mathcal{N}) \cap B_{k}\right| \geq \theta\right\} .
$$

Since $B_{k} \subset A_{k}$ for all $k$ it suffices to show that $B_{\infty}:=\bigcup_{k=0}^{\infty} B_{k}=B_{0} \cup U$.

The $B_{k}$ 's are vertically convex. To see why we first note that for $x \in U$ we have

$$
(x+\mathcal{N}) \cap\left(U^{\prime} \backslash U \backslash B_{0}\right)=\emptyset .
$$

In words, no $x \in U$ 'sees beneath' $B_{0}$. (13) follows from (6). For an arbitrary $x=$ $\left(x_{1}, x_{2}\right) \in U$ and $y=\left(y_{1}, y_{2}\right) \in U^{\prime} \backslash U \backslash B_{0}$ consider $P_{u, v}$ where $\exists\left(x_{1}, x_{2}^{\prime}\right) \in S_{u} \subset B_{0}$ and $\exists\left(y_{1}, y_{2}^{\prime}\right) \in S_{v} \subset B_{0}$. If $S_{u}$ and $S_{v}$ are not snug, then clearly $y \notin x+\mathcal{N}$. If $S_{u}$ and $S_{v}$ are snug, then by $(6) y \notin x+\mathcal{N}$.

It follows from (13) that if $\left(x_{1}, x_{2}\right),\left(x_{1}, x_{2}-1\right) \in U$, then

$$
\left|\left(\left(x_{1}, x_{2}\right)+\mathcal{N}\right) \cap B_{0}\right| \leq\left|\left(\left(x_{1}, x_{2}-1\right)+\mathcal{N}\right) \cap B_{0}\right|
$$

and

$$
\left(x_{1}, x_{2}\right) \in B_{1} \Rightarrow\left(x_{1}, x_{2}-1\right) \in B_{1} .
$$

So $\left\{(x, y) \in B_{1}: x=i\right\}$ is convex for every $i$ and no elements of $U \backslash B_{1}$ sees beneath $B_{1}$. Inductively applying these observations we get

$$
\left(x_{1}, x_{2}\right) \in B_{k} \cap U \Rightarrow\left(x_{1}, x_{2}-1\right) \in B_{k}
$$

for all $k$. So in order to describe $B_{k}$ it suffices to give the set $H^{k}=\left\{(x, y) \notin B_{k}\right.$ : $\left.(x, y-1) \in B_{k}\right\}$. Let $h^{k}(x)$ be the second coordinate of the element in $H^{k}$ with first coordinate $x$.

We first show that $B_{k+1} \neq B_{k}$ for all $k$. We consider the sum

$$
\sum_{(x, y) \in H^{k}}\left|((x, y)+\mathcal{N}) \cap B_{k}\right|
$$

In order to simplify this sum we define the following function for $l \leq i \leq r$ and $i-\rho \leq j \leq i+\rho$ :

$$
\psi(i, j)= \begin{cases}h^{k}(j)-h^{k}(i) & \text { if }-\rho \leq h^{k}(j)-h^{k}(i) \leq \rho+1, \\ -\rho & \text { if } h^{k}(j)-h^{k}(i)<-\rho \text { or } j \notin[l, r], \\ \rho+1 & \text { if } h^{k}(j)-h^{k}(i)>\rho+1 .\end{cases}
$$


For each $(x, y) \in H^{k}$, we have $\left|((x, y)+\mathcal{N}) \cap A_{k}\right|=(2 \rho+1) \rho+\sum_{j=x-\rho}^{x+\rho} \psi(x, j)$. Noting that $\psi(i, j)+\psi(j, i) \geq 0$ if $i, j \in[l, r]$ we have

$$
\begin{aligned}
\sum_{(x, y) \in H^{k}}\left|((x, y)+\mathcal{N}) \cap A_{k}\right|= & \sum_{i=l}^{r}\left[(2 \rho+1) \rho+\sum_{j=i-\rho}^{i+\rho} \psi(i, j)\right] \\
= & (r-l+1)(2 \rho+1) \rho+\sum_{i=l}^{r} \sum_{j=i-\rho}^{i+\rho} \psi(i, j) \\
\geq & (r-l+1)(2 \rho+1) \rho+\sum_{i=l}^{l+\rho-1} \sum_{j=i-\rho}^{l-1} \psi(i, j) \\
& +\sum_{i=r-\rho+1}^{r} \sum_{j=r+1}^{i+\rho} \psi(i, j) \\
\geq & (r-l+1)(2 \rho+1) \rho \\
& -2 \rho \sum_{j=l-\rho}^{l-1}(\rho+i-l+1) \\
= & (r-l+1)(2 \rho+1) \rho-\rho^{2}(\rho+1) \\
> & (r-l+1)[(2 \rho+1) \rho-1] .
\end{aligned}
$$

In the last inequality here we are using our assumption that $r-l>2 \rho^{3}$. From this calculation we conclude that for some $x \in[l, r],\left|\left(\left(x, h^{k}(x)\right)+\mathcal{N}\right) \cap B_{k}\right| \geq(2 \rho+1) \rho$, and $B_{k+1} \neq B_{k}$.

Let $C_{i}=\left\{(x, y) \in B_{\infty}: x=i\right\}$ (we call this column $i$ ). By (14) and our observation that $B_{k+1} \neq B_{k}$ for all $k$ it follows that there exists an $i$ such that $C_{i}=\left\{(x, y) \in U \cup B_{0}: x=i\right\}$. In words, there exists an infinite column. The proof is not yet complete because we do not yet know that all columns are infinite. Suppose that there is a finite column; suppose that $C_{i}=\left\{(x, y) \in U \cup B_{0}: x=i\right\}$ while $C_{i+1} \neq\left\{(x, y) \in U \cup B_{0}: x=i+1\right\}$. Let $m=\max \left\{y:(i+1, y) \in C_{i+1}\right\}$. Let $t$ be the first time at which $h^{t}(i)>m+\rho+1$. Note that $h^{t}(i+1)<h^{t}(i)$ which implies $\psi(i, j) \leq \psi(i+1, j)$ whenever both these functions are defined and $j \neq i, i+1$. Also note that $\psi(i+1, i)=\rho+1$ while $\psi(i, i+1)=-\rho$. Hence

$$
\begin{aligned}
\left|\left(\left(i+1, h^{t}(i+1)\right)+\mathcal{N}\right) \cap B_{t}\right| \geq & \\
-\left|\left(\left(i, h^{t}(i)\right)+\mathcal{N}\right) \cap B_{t}\right| \geq & \psi(i+1, i+1+\rho)-\psi(i, i-\rho) \\
& +\sum_{j=i-\rho+1}^{i+\rho} \psi(i+1, j)-\psi(x, j) \\
\geq & \psi(i+1, i+1+\rho)-\psi(i, i-\rho) \\
& +\psi(i+1, i)-\psi(i, i+1) \\
\geq & 0 .
\end{aligned}
$$

This is a contradiction.

To complete our discussion of the global properties of jagged blocks we show that all jagged blocks must be near $A_{0}$. In order to do this, we must exclude the tiny jagged blocks from consideration (i.e. we cannot prove - although it may be 
true - that jagged blocks must be near $A_{0}$ when the jagged blocks in question are very small). Let $\omega=\max \{\lfloor\rho / 2+1\rfloor,\lfloor\theta /(2 \rho+1)\rfloor\}$. For any $x \in \mathbf{Z}^{2}$ let

$$
C_{x}=\left\{x+\alpha e_{1}+\beta e_{2}:-\omega \leq \alpha, \beta \leq \omega\right\} \backslash\left\{x \pm \omega e_{1}, x \pm \omega e_{2}\right\}
$$

(this is a $(2 \omega+1) \times(2 \omega+1)$ square with corners removed). A jagged block $\mathcal{B}$ has a center $c(\mathcal{B})$ if we can choose $x \in \mathbf{Z}^{2}$ such that $c(\mathcal{B}):=C_{x} \subset \mathcal{B}$. Henceforth we consider only jagged blocks with centers.

We prove the following crucial lemma in the next section.

Low Density Lemma. If $\mathcal{B}$ is a jagged block with center, then there exists $b \in \mathcal{B}$ and $a \in A_{0}$ such that $\|b-a\|_{\infty} \leq \rho$.

We now turn our attention to the 'appropriate set' mentioned in the first paragraph of this section. We develop an alternate version of Lemma 2. We use the weight function given by (2) and (3), but, instead of letting $X=A_{n}$ for some large $n$, we let $X$ be the intersection of $A_{\infty}$ with some very large square (this intersection is the 'appropriate set'). To be precise, we use $X=\left\{x \in A_{\infty}:\|x\|_{\infty} \leq n\right\}$ where $n$ is chosen so that the following conditions hold:

(a) $A_{0} \subset X$;

(b) $|X| \gg\left|A_{0}\right|$; and

(c) $|X| \gg\left|\left\{x \in A_{\infty}: n-\rho<\|x\|_{\infty} \leq n+\rho\right\}\right|$.

Before considering the average of $\left|(x+\mathcal{N}) \cap A_{\infty}\right|$ over $X$ we verify that an $n$ satisfying these three conditions can be found.

Claim 5. There exists an $n$ satisfying $(a),(b)$, and $(c)$.

Proof. Conditions $(a)$ and $(b)$ are satisfied for all $n$ greater than some fixed $n_{0}$. To simultaneously achieve condition $(c)$ we will compare the density of $X$ around the boundary of the square with the density of $X$ over the whole square. Consider the sequences of densities

$$
d_{i}:=\frac{\left|\left\{x \in A_{\infty}:\|x\|_{\infty} \leq 2 \rho i\right\}\right|}{\left|\left\{x \in \mathbf{Z}^{2}:\|x\|_{\infty} \leq 2 \rho i\right\}\right|}
$$

and

$$
b_{i}:=\frac{\left|\left\{x \in A_{\infty}: 2 \rho i<\|x\|_{\infty} \leq 2 \rho(i+1)\right\}\right|}{\left|\left\{x \in \mathbf{Z}^{2}: 2 \rho i<\|x\|_{\infty} \leq 2 \rho(i+1)\right\}\right|} .
$$

We will show that

$$
b_{i}<4 d_{i} \text { for infinitely many } i \text {. }
$$

The existence of an $n$ satisfying (c) follows from (15) because, when $n=(2 i+1) \rho$ where both $b_{i}<4 d_{i}$ and $i$ is sufficiently large,

$$
\begin{aligned}
|X| & >(4 \rho i)^{2} d_{i} \\
& >4 \rho^{2} i^{2} b_{i} \\
& \gg 4(2 \rho)(4 \rho i+4 \rho) b_{i} \\
& >\left|\left\{x \in A_{\infty}: n-\rho<\|x\|_{\infty} \leq n+\rho\right\}\right| .
\end{aligned}
$$

We will say that $\left\{d_{i}\right\}$ is eventually increasing if there exists an $m_{0}$ such that $m>m_{0}$ implies $d_{m} \leq d_{m+1}$. If $\left\{d_{i}\right\}$ is eventually increasing, then $\left\{d_{i}\right\}$ converges to some limit density $d$. So, there exists some $m_{0}$ such that $i \geq m_{0}$ implies $d_{i}>d / 2$. If $\left\{d_{i}\right\}$ is eventually increasing, there exist infinitely many $i$ for which $b_{i}<2 d$. Therefore, when $\left\{d_{i}\right\}$ is eventually increasing we have (15). 
On the other hand, if $\left\{d_{i}\right\}$ is not eventually increasing, then there exist infinitely many $i$ such that $d_{i+1}<d_{i}$. For these $i$ we have $d_{i}>b_{i}$. Again, we have (15).

With these preliminaries in hand, we establish the alternate version of Lemma 2.

Claim 6. The average of $\left|(x+\mathcal{N}) \cap A_{\infty}\right|$ over the elements of $X$ is greater than $2 \theta+1 / 2$.

Proof. Choose $T$ so that $\left\{x \in A_{T}:\|x\|_{\infty} \leq n\right\}=X$ (since $X$ is finite such a $T$ exists). As in Lemma 2 we observe the evolution of the total weight, $W_{t}$, as $t$ goes from 0 to $T$. We have $W_{0} \leq\left|A_{0}\right|(2 \rho+1)^{2}$ and $W_{T}=0$. Again, we let $\Delta_{x}$ be the change in the total weight that results from the crystallization of $x$. Here we cannot claim, as we did in Lemma 2, that the sum over $X$ of $\Delta_{x}$ is $W_{T}-W_{0}$ because the crystallization of points $x$ with $n<\|x\|_{\infty} \leq n+\rho$ may cause an increase in the total weight. But, if we let $X^{\prime}=\left\{x \in A_{\infty}: n<\|x\|_{\infty} \leq n+\rho\right\}$, then we have

$$
W_{0}+\sum_{x \in X \backslash A_{0}} \Delta_{x}+\rho(2 \rho+1)\left|X^{\prime}\right| \geq W_{T} .
$$

Now, for any $x \in\left(A_{k+1} \backslash A_{k}\right) \cap X$ we have

$$
\Delta_{x}=\left|(x+\mathcal{N}) \cap X \cap\left(A_{T} \backslash A_{k+1}\right)\right|-\left|(x+\mathcal{N}) \cap A_{k}\right|
$$

and

$$
\begin{aligned}
\left|(x+\mathcal{N}) \cap A_{T}\right|= & \left|(x+\mathcal{N}) \cap\left(A_{T} \backslash A_{k+1}\right)\right|+\left|(x+\mathcal{N}) \cap\left(A_{k+1} \backslash A_{k}\right)\right| \\
& +\left|(x+\mathcal{N}) \cap A_{k}\right| \\
\geq & \left|(x+\mathcal{N}) \cap X \cap\left(A_{T} \backslash A_{k+1}\right)\right|+1+\left|(x+\mathcal{N}) \cap A_{k}\right| \\
= & 2\left|(x+\mathcal{N}) \cap A_{k}\right|+1+\Delta_{x} \\
\geq & 2 \theta+1+\Delta_{x} .
\end{aligned}
$$

Putting these two observations together we get

$$
\begin{aligned}
\sum_{x \in X}\left|(x+\mathcal{N}) \cap A_{T}\right| \geq & \sum_{x \in X \backslash A_{0}}\left|(x+\mathcal{N}) \cap A_{T}\right| \\
\geq & \left(|X|-\left|A_{0}\right|\right)(2 \theta+1)+\sum_{x \in X \backslash A_{0}} \Delta_{x} \\
\geq & \left(|X|-\left|A_{0}\right|\right)(2 \theta+1)+W_{T}-W_{0}-\rho(2 \rho+1)\left|X^{\prime}\right| \\
\geq & (2 \theta+1)|X|-(2 \theta+1)\left|A_{0}\right| \\
& -(2 \rho+1)^{2}\left|A_{0}\right|-\rho(2 \rho+1)\left|X^{\prime}\right| .
\end{aligned}
$$

Conditions $(b)$ and $(c)$ on our choice of $n$ imply that the sum of the last three terms in this final inequality will be greater than $-|X| / 2$.

We are now ready for the final step in the proof. We will again calculate the average of $\left|(x+\mathcal{N}) \cap A_{\infty}\right|$ over the elements of $X$, but now we will use our knowledge of how jagged blocks are situated in $A_{\infty}$. Let $H=\left\{x \in \mathbf{Z}^{2}:\|x\|_{\infty} \leq n\right.$ and $x \notin$ $\left.A_{\infty}\right\}$ (the set of holes). Let $H=\left\{h_{1}, \ldots, h_{m}\right\}$ be an arbitrary ordering of $H$. We define a map $\varphi: X \rightarrow H$ by $\varphi(x)=h_{i}$ if

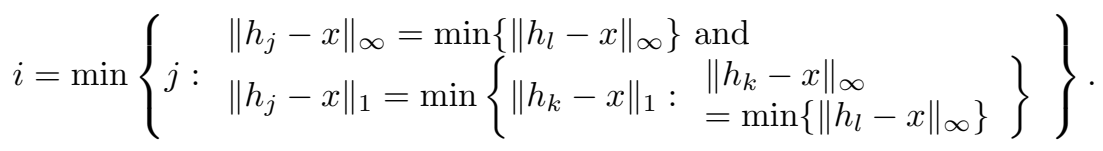


In words, $x$ is mapped to the nearest hole in $\ell_{\infty}$ distance where the first tie breaker is $\ell_{1}$ distance and the second tie breaker is the ordering on $H$. For most $x \in X$ we have $x \in C_{\varphi(x)}$; in fact, letting $X^{\prime}=\left\{x \in X: x \notin C_{\varphi(x)}\right\}$ we have

$$
\left|X^{\prime}\right| \leq\left|\left\{x \in X: n-\rho<\left\|x_{\infty}\right\| \leq n\right\}\right|+\left(2 \rho^{3}\right)^{2}\left|A_{0}\right|(2 \rho+1)^{2} .
$$

Proof. Suppose $\varphi(x)=h$ and $x \notin C_{h}$. Since $x \notin C_{h}$, either $\|x-h\|_{\infty}>\omega$ or $x \in$ $\left\{h \pm \omega e_{1} \pm \omega e_{2}\right\}$. If $\|x-h\|_{\infty}>\omega$, then $\varphi(x)=h$ implies $B_{\infty}\left(x,\|x-h\|_{\infty}-1\right) \cap H=\emptyset$ and $C_{x} \cap H=\emptyset$. If $x \in\left\{h \pm \omega e_{1} \pm \omega e_{2}\right\}$, then $\varphi(x)=h$ implies $C_{x} \cap H=\emptyset$. If $C_{x} \cap H=\emptyset$, then either $x$ is in a jagged block with center or $\|x\|_{\infty}>n-\rho$. By the Low Density Lemma there are at most $\left|A_{0}\right|(2 \rho+1)^{2}$ jagged blocks in $X$, and by Lemma 4 each of these jagged blocks has cardinality at most $\left(2 \rho^{3}\right)^{2}$.

We will now consider the average of $\left|(x+\mathcal{N}) \cap A_{\infty}\right|$ over sets of the form $\varphi^{-1}(h)$. According to (16), when we apply the following claim, the proof of which is given in section 5 , to all $h \in H$ we see that the average of $\left|(x+\mathcal{N}) \cap A_{T}\right|$ over most of $X$ is smaller than $2 \theta$.

Local Averaging Claim. The average of $\left|(x+\mathcal{N}) \cap A_{\infty}\right|$ over the elements of $\varphi^{-1}(h) \cap C_{h}$ is less than $2 \theta$.

So the average of $\left|(x+\mathcal{N}) \cap A_{\infty}\right|$ over most of the elements of $X$ is strictly less than $2 \theta$ while the average over all of $X$ of $\left|(x+\mathcal{N}) \cap A_{\infty}\right|$ must be greater than $2 \theta+1 / 2$. This should give a contradiction.

To do this rigorously consider the sum

$$
\begin{aligned}
\sum_{x \in X}\left|(x+\mathcal{N}) \cap A_{\infty}\right|= & \sum_{h \in H} \sum_{x \in \varphi^{-1}(h)}\left|(x+\mathcal{N}) \cap A_{\infty}\right| \\
= & \sum_{h \in H} \sum_{x \in \varphi^{-1}(h) \cap C_{h}}\left|(x+\mathcal{N}) \cap A_{\infty}\right| \\
& +\sum_{x \in X^{\prime}}\left|(x+\mathcal{N}) \cap A_{\infty}\right| \\
\leq & 2 \theta|X|+\left|X^{\prime}\right|(2 \rho+1)^{2} .
\end{aligned}
$$

By conditions $(b)$ and $(c)$ on the choice of $n$, we have $\left|X^{\prime}\right| \ll|X|$ and the average of $\left|(x+\mathcal{N}) \cap A_{\infty}\right|$ over the elements of $X$ is less than $2 \theta+1 / 2$. This contradicts Claim 6.

\section{Proof of the Low Density Lemma}

We prove the Low Density Lemma indirectly. Assume $\mathcal{B}$ is a jagged block which satisfies $d_{\infty}\left(A_{0}, \mathcal{B}\right):=\min \left\{\|x-y\|_{\infty}: x \in \mathcal{B}, y \in A_{0}\right\}>\rho$. Let $t=\min \left\{i: A_{i} \cap \mathcal{B} \neq\right.$ $\emptyset\}$ and let $x \in A_{t} \cap \mathcal{B}$. In words, $x$ is one of the first elements of $\mathcal{B}$ to crystallize. Since $d_{\infty}\left(A_{0}, \mathcal{B}\right)>\rho, x \notin A_{0}$ and $\left|(x+\mathcal{N}) \cap A_{t-1}\right| \geq \theta$. Now, suppose $p$ is a peg of $\mathcal{B}$ that lies near $x$ (a large part of the work we do here is in finding this peg). The neighborhood of $p$ will contain many of the at least $\theta$ elements of $(x+\mathcal{N}) \cap A_{t-1}$ as well as at least (and in some cases much more than) $\rho^{2}$ elements of $\mathcal{B}$. There are also elements of $A_{\infty} \backslash A_{t-1} \backslash \mathcal{B}$ that are seen by $p$. We will show that for some peg $p$ near $x$ we have

$$
\begin{gathered}
\left|(p+\mathcal{N}) \cap\left((x+\mathcal{N}) \cap A_{t-1}\right)\right|+|(p+\mathcal{N}) \cap \mathcal{B}| \\
+\left|(p+\mathcal{N}) \cap\left(A_{\infty} \backslash A_{t-1} \backslash \mathcal{B}\right)\right| \geq \theta .
\end{gathered}
$$


Since $\left|(p+\mathcal{N}) \cap A_{\infty}\right|$ equals the left-hand side of $(17)$, it contradicts $p \notin A_{\infty}$.

We divide the argument into cases depending on how $x$ is situated in $\mathcal{B}$. For most of these cases we use only the first two terms in (17). In some of the cases, however, we will resort to using elements of $A_{\infty} \backslash A_{t-1} \backslash \mathcal{B}$.

Why should there be any elements of $(p+\mathcal{N}) \cap\left(A_{\infty} \backslash A_{t-1} \backslash \mathcal{B}\right)$ ? Suppose $z \in$ $(x+\mathcal{N}) \cap A_{t-1}$ and $\|z-w\|_{1} \leq|(z+\mathcal{N}) \cap \mathcal{B}| /(2 \rho+1)$. Using the fact $z \notin A_{0}$ (which follows from the assumption $\left.d_{\infty}\left(A_{0}, \mathcal{B}\right)>\rho\right)$ and noting that $|(z+\mathcal{N}) \backslash(w+\mathcal{N})| \leq$ $\|z-w\|_{1}(2 \rho+1)$ we have

$$
\begin{aligned}
\left|(w+\mathcal{N}) \cap A_{\infty}\right| & \geq\left|(z+\mathcal{N}) \cap A_{\infty}\right|-|(z+\mathcal{N}) \backslash(w+\mathcal{N})| \\
& \geq\left|(z+\mathcal{N}) \cap A_{\infty}\right|-|| z-w \|_{1}(2 \rho+1) \\
& \geq\left(\left|(z+\mathcal{N}) \cap A_{t-1}\right|+|(z+\mathcal{N}) \cap \mathcal{B}|\right)-|(z+\mathcal{N}) \cap \mathcal{B}| \\
& \geq \theta
\end{aligned}
$$

We have shown

$$
z \in(x+\mathcal{N}) \cap A_{t-1} \Rightarrow\left\{w:\|z-w\|_{1} \leq \frac{|(z+\mathcal{N}) \cap \mathcal{B}|}{(2 \rho+1)}\right\} \subset A_{\infty} .
$$

To see how this implies the existence of elements of $(p+\mathcal{N}) \cap\left(A_{\infty} \backslash A_{t-1} \backslash \mathcal{B}\right)$ first note that we expect $M:=((p+\mathcal{N}) \cap(x+\mathcal{N})) \backslash \mathcal{B}$ to contain both elements of $A_{t-1}$ (since $\left|(x+\mathcal{N}) \cap A_{t-1}\right|$ is large) and elements of $\mathbf{Z}^{2} \backslash A_{\infty}$ (since $\left|(p+\mathcal{N}) \cap A_{\infty}\right|$ is small). (18) then implies the existence of a 'region' of elements of $\left(A_{\infty} \backslash A_{t-1}\right) \cap M$ that lies between $A_{t-1} \cap M$ and $\left(\mathbf{Z}^{2} \backslash A_{\infty}\right) \cap M$. We believe that this region is rather large, but we don't see any useful, general lower bound on its size. So we appeal to (18) only sparingly in what follows. However, the existence of this region is a heuristic reason for believing that none of the following calculations are tight (i.e. in each case we find a peg $p$ where $\left|(p+\mathcal{N}) \cap A_{\infty}\right|$ is actually much larger than $\left.\theta\right)$.

Before considering the cases, we establish some definitions and notational conventions. For ease of notation we assume $x$ is the origin (so in particular we can write $\mathcal{N}$ for $x+\mathcal{N})$. For $z=\left(z_{1}, z_{2}\right), y=\left(y_{1}, y_{2}\right) \in \mathbf{Z}^{2}$ let $[z, y]:=\left\{\left(w_{1}, w_{2}\right) \in \mathbf{Z}^{2}\right.$ : $w_{1}=\lambda_{1} z_{1}+\left(1-\lambda_{1}\right) y_{1}$ and $w_{2}=\lambda_{2} z_{2}+\left(1-\lambda_{2}\right) y_{2}$ where $\left.0 \leq \lambda_{1}, \lambda_{2} \leq 1\right\}$; that is $[z, y]$ is the rectangle in $\mathbf{Z}^{2}$ with corners $z$ and $y$. Let $\partial \mathcal{N}=\left\{z:\|z\|_{\infty}=\rho\right\}$. We will refer to $\partial \mathcal{N}$ as the boundary of $\mathcal{N}$. For $\alpha, \beta \in\{-1,1\}$ let $\left.Q_{\alpha, \beta}=[(\alpha, \beta),(\alpha \rho, \beta \rho))\right]$ and $\bar{Q}_{\alpha, \beta}=[(0,0),(\alpha \rho, \beta \rho)]$; we will use the terms open quadrant and closed quadrant, respectively, for these sets. Our analysis of how $x$ is situated in $\mathcal{B}$ will depend heavily on the set $\left.\mathcal{B}\right|_{\mathcal{N}}:=\bigcup\left\{S_{z}: S_{z} \subset \mathcal{B} \cap \mathcal{N}\right\}$. We say $\left.S_{z} \subset \mathcal{B}\right|_{\mathcal{N}}$ is a corner square if the origin is in $S_{z}$ and there exist pegs $p, q \in \mathcal{N}$ and $\alpha, \beta \in\{-1,1\}$ such that $p \in S_{z+\alpha e_{1}}$ and $q \in S_{z+\beta e_{2}}$ (i.e. there are pegs adjacent to two intersecting sides of $S_{z}$ ).

For the purpose of describing the cases we will need the following important definitions. A corner square $\left.S_{z} \subset \mathcal{B}\right|_{\mathcal{N}}$ will be called an exposed corner if there exist pegs $p, q \in \mathcal{N}$ lying in opposite open quadrants - to be precise, $p \in Q_{\delta, \gamma}$ and $q \in Q_{-\delta,-\gamma}$ for some $\delta, \gamma \in\{-1,1\}-$ and $\alpha, \beta \in\{-1,1\}$ such that $p \in S_{z+\alpha e_{1}}$ and $q \in S_{z+\beta e_{2}}$. Roughly speaking, $\left.\mathcal{B}\right|_{\mathcal{N}}$ has an exposed corner if $x$ is close to the edge of $\mathcal{B}$. A corner peg $p$ of $\mathcal{B}$ will be called an outside corner peg if $p \in \mathcal{N}, S_{\mathcal{B}, p}$ is not contained in $\mathcal{N}$ and $p$ is adjacent to $\left.\mathcal{B}\right|_{\mathcal{N}}$. Outside corner pegs are important only because they allow us to construct a list of cases that is exhaustive.

We divide the proof into five cases. In the first and most important case we handle exposed corners (i.e. we handle $x$ 's that are close to the edge of $\mathcal{B}$ ). A reader who is interested in only a heuristic justification of the Low Density Lemma 
might read only this case; it seems heuristically obvious to the author that there is no way the crystal can 'jump' to the middle of $\mathcal{B}$, but, unfortunately, the author has found no direct way to handle the other cases. Cases $2-5$ contain every possible positioning of $x$ in $\mathcal{B}$. Case 1 is important in this analysis because we often reduce these other cases to Case 1 . The four cases that cover every possible positioning of $x$ in $\mathcal{B}$ are:

2. $\left.\mathcal{B}\right|_{\mathcal{N}}$ contains an open quadrant.

3. There exists an outside corner peg.

4. $\left.\partial \mathcal{N} \cap \mathcal{B}\right|_{\mathcal{N}}$ contains a set of the form $\left[w, w+2 \omega e_{1}\right]$ or a set of the form $\left[w, w+2 \omega e_{2}\right]$.

5. $c(\mathcal{B}) \subset \mathcal{N}$.

To see how these four cases cover everything we begin with the following lemma. For $v \in\left\{ \pm e_{1}, \pm e_{2}\right\}$ let $\partial_{v} \mathcal{N}=\{w \in \partial \mathcal{N}: w-2 \rho v \in \partial \mathcal{N}\}$ and $\partial_{v} \mathcal{B}=\left\{u \in \partial_{v} \mathcal{N}\right.$ : $\exists n \in \mathbf{N}$ and $w \in \mathcal{B}$ such that $u+n v=w\}$ (in some sense $\partial_{v} \mathcal{B}$ is a projection of $\mathcal{B}$ onto $\partial \mathcal{N})$.

Lemma 7. If $Q_{-1,-1}, Q_{1,-1} \not \subset \mathcal{B}$ and $\left.\partial_{-e_{2}} \mathcal{B} \not \subset \mathcal{B}\right|_{\mathcal{N}} \cap \partial_{-e_{2}} \mathcal{N}$, then there is an outside corner peg (analogous statements hold for $\partial_{e_{1}} \mathcal{B}, \partial_{-e_{1}} \mathcal{B}$ and $\partial_{e_{2}} \mathcal{B}$ ).

Proof. First note that (4) implies $L:=\left.\mathcal{B}\right|_{\mathcal{N}} \cap \partial_{-e_{2}} \mathcal{N}$ consists of a single interval. Say $L=[(\alpha,-\rho),(\beta,-\rho)]$. If $\partial_{-e_{2}} \mathcal{B}$ is not contained in $L$, then there exists $S_{z:=\left(z_{1}, z_{2}\right)} \subset$ $\mathcal{B}$ with $z_{2}<-\rho$ and $-\rho \leq z_{1} \leq 1$ such that $\left.S_{\left(z_{1},-\rho\right)} \not \subset \mathcal{B}\right|_{\mathcal{N}}$. Assume without loss of generality that $z_{1}<\alpha$. The basis vectors of $P_{(\alpha,-\rho), z}$ are $-e_{1}$ and $-e_{2}$. By the definition of $\left.\mathcal{B}\right|_{\mathcal{N}}, S_{(\alpha,-\rho)-e_{1}} \not \subset \mathcal{B}$. By $(12)$ there exists $p \in[(\alpha-1,-\rho),(\alpha-1,-1)]$ such that $p \notin A_{\infty}$ and $p-e_{2} \in \mathcal{B}$. Since $p+e_{1}$ is clearly in $\left.\mathcal{B}\right|_{\mathcal{N}}, p$ is an outside corner peg.

Now consider the location of $c(\mathcal{B})$. If $\left.\mathcal{B}\right|_{\mathcal{N}}$ contains no open quadrant and there exists no outside corner peg (i.e. if Cases 2 and 3 do not hold), then by Lemma 7

$$
\left.\mathcal{B} \subset \mathcal{B}\right|_{\mathcal{N}} \cup\left(\left.\bigcup_{v \in\left\{ \pm e_{1}, \pm e_{2}\right\}} \mathcal{B}\right|_{\mathcal{N}} \cap \partial_{v} \mathcal{N}+\mathbf{N} v\right)
$$

If we also have $c(\mathcal{B}) \not \subset \mathcal{N}$ (i.e. if Case 5 does not hold), then $\left.c(\mathcal{B}) \subset \mathcal{B}\right|_{\mathcal{N}} \cap\left(\partial_{v} \mathcal{N}+\mathbf{Z} v\right)$ for some $v \in\left\{ \pm e_{1}, \pm e_{2}\right\}$. This implies that we are in Case 4.

We now consider the cases. Throughout this analysis we are always assuming that previous cases do not hold; when considering Case $i$ we are assuming that we are not in Case $j$ for $j<i$.

Case 1. $\left.\mathcal{B}\right|_{\mathcal{N}}$ has an exposed corner.

This is the crucial case. From a heuristic point of view, all reasonable arrangements for 'breaching' a jagged block fall into this case. It is also important to assume $\left.\mathcal{B}\right|_{\mathcal{N}}$ has no exposed corner for the cases that follow this one; after this case we have

$$
\left.S_{z} \subset \mathcal{B}\right|_{\mathcal{N}} \text { is a corner square } \Rightarrow \exists \operatorname{peg} p \in \bar{Q}_{\alpha, \beta} \text { adjacent to } S_{z}
$$

where $\alpha$ and $\beta$ are as in the definition of a corner square. The following claim is one illustration of the utility of (19): the proof of the Low Density Lemma follows easily from (19) for $\rho^{2}<\theta \leq 4 / 3 \rho^{2}$. 
Claim 8. If $\left.\mathcal{B}\right|_{\mathcal{N}}$ has no exposed corners and $\rho^{2}<\theta \leq 4 / 3 \rho^{2}$, then there exists $p \in\left(\mathbf{Z}^{2} \backslash A_{\infty}\right) \cap \mathcal{N}$ such that $\left|(p+\mathcal{N}) \cap A_{\infty}\right| \geq \theta$.

Proof. Let $\alpha, \beta \in\{-1,1\}$. Let $S_{z}$ be a square in $\left.\mathcal{B}\right|_{\mathcal{N}}$ for which $S_{z}+\alpha e_{1}, S_{z}+\beta e_{2} \not \subset$ $\left.\mathcal{B}\right|_{\mathcal{N}}$. Either $S_{z}=Q_{\alpha, \beta}$ or $S_{z}$ is adjacent to a peg $p_{\alpha, \beta} \in \mathcal{N}$. In the later situation (19) implies $p_{\alpha, \beta} \in \bar{Q}_{\alpha, \beta}$. Let $P=\left\{p_{\alpha, \beta}: \alpha, \beta \in\{-1,1\}\right.$ for which $p_{\alpha, \beta}$ exists $\}$. Note that $P \neq \emptyset$ (if $P=\emptyset$, then by $(7) \mathcal{N} \subset \mathcal{B}$, which contradicts $\left|\mathcal{N} \cap A_{t-1}\right| \geq \theta$ ).

Consider an arbitrary $w$ not equal to $x$ in $\mathcal{N}$. If $w$ is contained in the open quadrant $Q_{\alpha, \beta}$, then either $w \in p_{\alpha, \beta}+\mathcal{N} \subset \bigcup_{p \in P}(p+\mathcal{N})$ or $w \in \mathcal{B}$. If $w$ is contained in no open quadrant (i.e. if $w$ lies on the coordinate axes), then $w \in \bar{Q}_{\alpha, \beta} \cap \bar{Q}_{\alpha^{\prime}, \beta^{\prime}}$ for some $\alpha, \beta, \alpha^{\prime}, \beta^{\prime} \in\{-1,1\}$. If either $p_{\alpha, \beta}$ or $p_{\alpha^{\prime}, \beta^{\prime}}$ exist, then $w \in \bigcup_{p \in P}(p+\mathcal{N})$. If neither $p_{\alpha, \beta}$ nor $p_{\alpha^{\prime}, \beta^{\prime}}$ exist, then $Q_{\alpha, \beta}, Q_{\alpha^{\prime}, \beta^{\prime}} \subset \mathcal{B}$ which implies, by (7), that $w \in \mathcal{B}$. So $\mathcal{N} \backslash \mathcal{B} \subset \bigcup_{p \in P}(p+\mathcal{N})$.

This implies that for some $p \in P$ we have $\left|(p+\mathcal{N}) \cap A_{t-1}\right| \geq \theta / 4$. But, since $p$ is a peg, we also have $\left|(p+\mathcal{N}) \cap\left(A_{\infty} \backslash A_{t-1}\right)\right| \geq \rho^{2}$. Putting these two observations together we get $\left|(p+\mathcal{N}) \cap A_{\infty}\right| \geq \rho^{2}+\theta / 4 \geq \theta$.

We now proceed with the proof for Case 1 . Let $S_{z}$ be an exposed corner with $p$ and $q$ as in the definition of an exposed corner. We assume, without loss of generality, that $p \in Q_{1,-1}$ and $q \in Q_{-1,1}$. Let $z=\left(-z_{1},-z_{2}\right), p=\left(p_{1},-p_{2}\right)$, and $q=\left(-q_{1}, q_{2}\right)$ (so, we have $z_{1}, z_{2} \geq 0$ and $p_{1}, p_{2}, q_{1}, q_{2}>0$ ). It follows from our definitions that $z_{1} \geq q_{1}$ and $z_{2} \geq p_{2}$ (i.e. $p$ is on the right edge of $S_{z}$ and $q$ is on the top edge of $S_{z}$ ). Either $p$ or $q$ sees many of the elements of $A_{t-1} \cap \mathcal{N}$ : since $|\mathcal{N} \backslash((p+\mathcal{N}) \cup(q+\mathcal{N}))|=p_{1} q_{2}+p_{2} q_{1}$ we have

$$
\begin{aligned}
\left|(p+\mathcal{N}) \cap A_{t-1}\right|+\left|(q+\mathcal{N}) \cap A_{t-1}\right| & \geq\left|((p+\mathcal{N}) \cup(q+\mathcal{N})) \cap A_{t-1}\right| \\
& \geq \theta-p_{1} q_{2}-p_{2} q_{1} .
\end{aligned}
$$

At the same time, since $S_{z} \subset \mathcal{B} \subset A_{\infty} \backslash A_{t-1}$ and both $p$ and $q$ see all of $S_{z}$, we have

$$
\left|(p+\mathcal{N}) \cap A_{\infty} \backslash A_{t-1}\right|+\left|(q+\mathcal{N}) \cap A_{\infty} \backslash A_{t-1}\right| \geq 2 \rho^{2} .
$$

But, these are not the only elements of $\mathcal{B}$ (and, therefore, of $A_{\infty} \backslash A_{t-1}$ ) seen by either $p$ or $q$. In each of the following subcases we strengthen (21) by finding a set (or collection of disjoint sets) that lies in $((p+\mathcal{N}) \cup(q+\mathcal{N})) \cap \mathcal{B} \backslash S_{z}$ and thereby arrive at a contradiction.

Subcase 1.1. Either $p$ or $q$ is a corner peg.

Assume without loss of generality that $p$ is a corner peg. Note that by (10) we must have $p-e_{2} \in \mathcal{B}$. The neighborhood of $p$ contains both $S_{z}$ and $S_{\mathcal{B}, p}$. Since $\left|S_{\mathcal{B}, p} \backslash S_{z}\right|=\rho\left(q_{2}+p_{2}\right)$, we get

$$
\left|(p+\mathcal{N}) \cap A_{\infty} \backslash A_{t-1}\right|+\left|(q+\mathcal{N}) \cap A_{\infty} \backslash A_{t-1}\right| \geq 2 \rho^{2}+\rho\left(q_{2}+p_{2}\right) .
$$


Adding this to what we get from (20) we have

$$
\begin{aligned}
\left|(p+\mathcal{N}) \cap A_{\infty}\right|+\left|(q+\mathcal{N}) \cap A_{\infty}\right| \geq & |(p+\mathcal{N}) \cap \mathcal{B}|+|(q+\mathcal{N}) \cap \mathcal{B}| \\
& +\left|((p+\mathcal{N}) \cup(q+\mathcal{N})) \cap A_{t-1}\right| \\
\geq & 2 \rho^{2}+\rho\left(p_{2}+q_{2}\right)+\theta-p_{1} q_{2}-p_{2} q_{1} \\
= & \theta+2 \rho^{2}+q_{2}\left(\rho-p_{1}\right)+p_{2}\left(\rho-q_{1}\right) \\
\geq & \theta+\left(2 \rho^{2}+\rho\right)+\left(\rho-q_{1}-p_{1}\right) \\
\geq & \theta+\rho(2 \rho+1) \\
\geq & 2 \theta .
\end{aligned}
$$

Hence, either $p$ or $q$ sees at least $\theta$ elements of $A_{\infty}$. This, of course, is a contradiction.

Subcase 1.2. Neither $p$ nor $q$ is a corner peg.

In this case we use the fact that $\mathcal{B}$ has a center to strengthen (21). In order to describe $(p+\mathcal{N}) \cap \mathcal{B}$ and $(q+\mathcal{N}) \cap \mathcal{B}$ we first establish some simple claims concerning $\mathcal{B}$.

Claim 9. We may assume

$$
q_{2}-1=\max \left\{y_{2}: \exists\left(y_{1}, y_{2}\right) \in \mathcal{B}\right\}
$$

and

$$
p_{1}-1=\max \left\{y_{1}: \exists\left(y_{1}, y_{2}\right) \in \mathcal{B}\right\} .
$$

Proof. Suppose there exists $y=\left(y_{1}, y_{2}\right) \in \mathcal{B}$ with $y_{2} \geq q_{2}$. We have $y \in S_{w}$ for some $S_{w} \subset \mathcal{B}$. The basis vectors of $P_{z, w}$ are $-e_{1}$ and $e_{2}$ (they can't be $e_{1}$ and $e_{2}$ because neither $S_{z}+e_{1}$ nor $S_{z}+e_{2}$ are subsets of $\mathcal{B}$ ). By assumption $S_{x}+e_{2} \not \subset \mathcal{B}$. So, by (12) there is a corner square in $S_{x}+e_{2}$. We can let $q$ be this corner square and appeal to Subcase 1.1.

Let $C=S_{z}-\rho e_{2}$. For any $y \in C$ let $y^{\prime}=y-\rho e_{1}+\rho e_{2}$, and let $C^{\prime}=\left\{y^{\prime}: y \in C\right\}$ (note that $C^{\prime}$ is also known as $S_{z}-\rho e_{1}$ ).

Claim 10. If $y \in C$ and neither $y=\left(y_{1}, y_{2}\right)$ nor $y^{\prime}=\left(y_{1}^{\prime}, y_{2}^{\prime}\right)$ is in $\mathcal{B}$, then $\mathcal{B} \subset R:=\left[\left(y_{1}^{\prime}+1, y_{2}+1\right),\left(p_{1}-1, q_{2}-1\right)\right]\left(R\right.$ is the rectangle bounded by $\left.\left\{y, y^{\prime}, q, p\right\}\right)$.

Proof. Suppose $\mathcal{B}$ is not contained in $R$. Then there exists $S_{v} \subset \mathcal{B}$ with $S_{v} \cap R=$ $\rho(\rho-1)$. If this is the case then, appealing to Claim 9, either $v_{1}=y_{1}^{\prime}$ and $y^{\prime} \in S_{v}$ or $v_{2}=y_{2}$ and $y \in S_{v}$. Hence, either $y \in \mathcal{B}$ or $y^{\prime} \in \mathcal{B}$.

Claim 11. If $y \in C \cap \mathcal{B}$ or $y \in C^{\prime} \cap \mathcal{B}$, then $[y, z] \subset \mathcal{B}$.

Proof. Suppose $y \in C \cap \mathcal{B}$ and let $S_{w}$ be some $\rho \times \rho$ square in $\mathcal{B}$ containing $y$. Clearly, $S_{w}$ and $S_{z}$ are tight. Since $[y, z] \subset S_{w} \cup S_{z} \cup R_{w, z}^{c}$, it follows from (7) that $[y, z] \subset \mathcal{B}$.

Now, assume without loss of generality that $q_{2}+p_{2} \leq q_{1}+p_{1}$. Of course the argument won't go quietly, and we must, once again, divide and conquer.

Subcase 1.2.1. $q_{2}+p_{2}+\rho \leq 2 \omega+1$. 


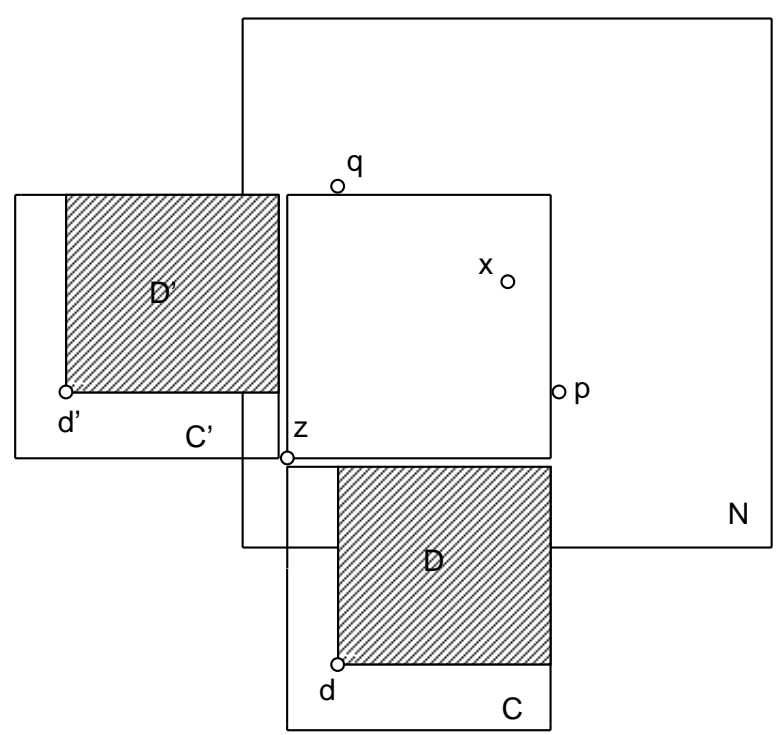

Figure 1. Subcase 1.2.1

We now strengthen (21) by finding two disjoint sets contained in $((p+\mathcal{N}) \cup(q+$ $\mathcal{N})) \cap \mathcal{B} \backslash S_{z}$

Let $D=\left\{y \in C: y \in p+\mathcal{N}\right.$ and $\left.y^{\prime} \in q+\mathcal{N}\right\}$. Let $D^{\prime}=D-\rho e_{1}+\rho e_{2}=\left\{y^{\prime}:\right.$ $y \in D\}$. Note that $D \subset p+\mathcal{N}$ and $D^{\prime} \subset q+\mathcal{N}$. A picture of these regions is given in Figure 1.

Assume for the sake of contradiction that $y=\left(y_{1}, y_{2}\right), y^{\prime}=\left(y_{1}^{\prime}, y_{2}^{\prime}\right) \notin \mathcal{B}$ for some $y \in D$. This implies, by Claim 10 , that $\mathcal{B} \subset\left[\left(y_{1}^{\prime}+1, y_{2}+1\right),\left(p_{1}-1, q_{2}-1\right)\right]$. It follows that $\max \left\{\left|u_{2}-v_{2}\right|:\left(u_{1}, u_{2}\right),\left(v_{1}, v_{2}\right) \in \mathcal{B}\right\}<q_{2}+p_{2}+\rho-1 \leq 2 \omega$ which contradicts the fact that $\mathcal{B}$ has a center. So for every $y \in D$ either $y$ or $y^{\prime}$ is in $\mathcal{B}$. Hence (see $(21)),|(q+\mathcal{N}) \cap \mathcal{B}|+|(p+\mathcal{N}) \cap \mathcal{B}| \geq 2 \rho^{2}+|D|$.

To further strengthen (21) consider $d:=\left(-q_{1},-p_{2}-\rho\right)$ ( $d$ is the bottom left hand element of $D)$. Note that $[d, z] \subset p+\mathcal{N}$ and that $\left[d^{\prime}, z\right] \subset q+\mathcal{N}$. We've already determined that either $d$ or $d^{\prime}$ is in $\mathcal{B}$. Claim 11 then implies that either $\left[d-e_{1}, z-e_{2}\right]$ or $\left[d^{\prime}-e_{2}, z-e_{1}\right]$ is in $\mathcal{B}$ (note that $\left[d-e_{1}, z-e_{2}\right] \subset[d, z]$ and $\left.\left[d^{\prime}-e_{2}, z-e_{1}\right] \subset\left[d^{\prime}, z\right]\right)$. Since

$$
\begin{aligned}
\left|\left[d^{\prime}-e_{2}, z-e_{1}\right]\right| & =\left(p_{1}+q_{1}\right)\left(\rho-p_{2}-q_{2}\right) \\
& \geq\left(p_{2}+q_{2}\right)\left(\rho-p_{1}-q_{1}\right) \\
& =\left|\left[d-e_{1}, z-e_{2}\right]\right|
\end{aligned}
$$

we now have

$$
\begin{aligned}
|(q+\mathcal{N}) \cap \mathcal{B}|+|(p+\mathcal{N}) \cap \mathcal{B}| \geq & 2 \rho^{2}+|D|+\left|\left[d-e_{1}, z-e_{2}\right]\right| \\
= & 2 \rho^{2}+\left(p_{1}+q_{1}\right)\left(p_{2}+q_{2}\right) \\
& +\left(p_{2}+q_{2}\right)\left(\rho-p_{1}-q_{1}\right) \\
= & 2 \rho^{2}+\rho\left(p_{2}+q_{2}\right) .
\end{aligned}
$$




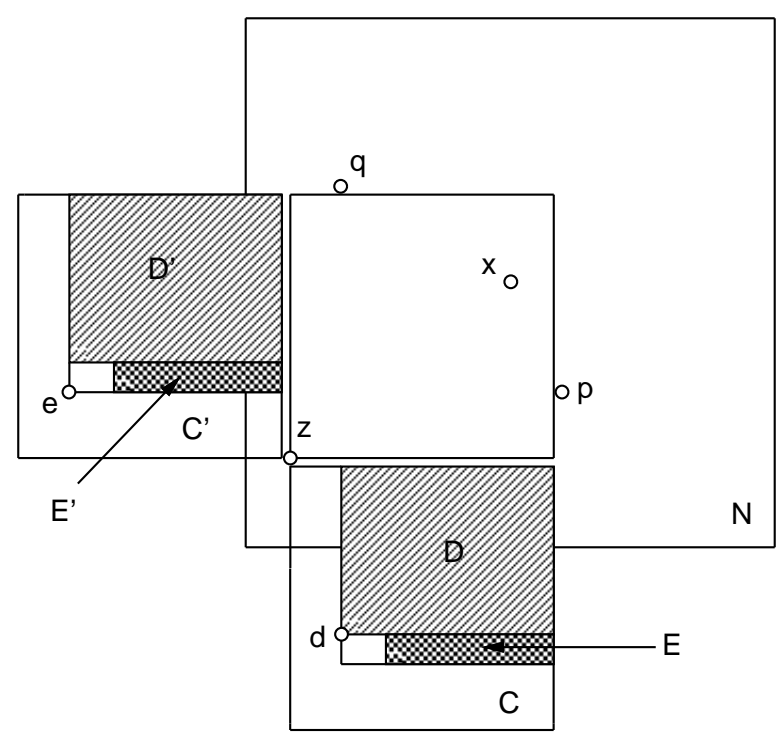

FiguRE 2. Subcase 1.2.2

This is our fully strengthened (21). Adding it to what we get from (20) we have

$$
\begin{aligned}
\left|(q+\mathcal{N}) \cap A_{\infty}\right|+\left|(p+\mathcal{N}) \cap A_{\infty}\right| & \geq 2 \rho^{2}+\rho\left(p_{2}+q_{2}\right)+\theta-p_{1} q_{2}-p_{2} q_{1} \\
& =\theta+2 \rho^{2}+q_{2}\left(\rho-p_{1}\right)+p_{2}\left(\rho-q_{1}\right) \\
& \geq \theta+\left(2 \rho^{2}+\rho\right)+\left(\rho-q_{1}-p_{1}\right) \\
& \geq \theta+\rho(2 \rho+1) \\
& \geq 2 \theta .
\end{aligned}
$$

Subcase 1.2.2. $q_{2}+p_{2}+\rho>2 \omega+1$.

Once again we strengthen (21) by finding two disjoint sets which lie in $((p+$ $\mathcal{N}) \cup(q+\mathcal{N})) \cap \mathcal{B} \backslash S_{z}$.

Let $D=\left\{y=\left(y_{1}, y_{2}\right) \in C: y \in p+\mathcal{N}, y^{\prime} \in q+\mathcal{N}, q_{2}-y_{2} \leq 2 \omega+1\right\}$ and let $E=\left\{y=\left(y_{1}, y_{2}\right) \in C: y \in p+\mathcal{N}, y^{\prime} \in q+\mathcal{N}, p_{1}-y_{1}^{\prime} \leq 2 \omega+1\right\} \backslash D$. Let $D^{\prime}=D-\rho e_{1}+\rho e_{2}=\left\{d^{\prime}: d \in D\right\}$ and $E^{\prime}=E-\rho e_{1}+\rho e_{2}=\left\{e^{\prime}: e \in E\right\}$. A picture of this is given in Figure 2. Note that $E \neq \emptyset$ precisely because $q_{2}+p_{2}+\rho>2 \omega+1$.

By Claim 10, for every $y \in D \cup E$ either $y$ or $y^{\prime}$ is in $\mathcal{B}$ (the proof of this follows as it did in Subcase 1.2.1). Hence, $|(q+\mathcal{N}) \cap \mathcal{B}|+|(p+\mathcal{N}) \cap \mathcal{B}| \geq 2 \rho^{2}+|D|+|E|$.

Let $d=\left(-q_{1}, q_{2}-2 \omega-1\right)$ (this is the bottom left hand corner of $\left.D\right)$ and let $e=\left(-q_{1}-\rho,-p_{2}\right)$. Due to Claim 11 (and the fact that $e \in\left[d^{\prime}, z\right]$ ) either $\left[d-e_{1}, z-e_{2}\right] \subset \mathcal{B}$ or $\left[e-e_{2}, z-e_{1}\right] \subset \mathcal{B}$. Since

$$
\begin{aligned}
\left|\left[e-e_{2}, z-e_{1}\right]\right| & =\left(p_{1}+q_{1}\right)\left(\rho-p_{2}-q_{2}\right) \\
& \geq(2 \omega+1-\rho)\left(\rho-p_{1}-q_{1}\right) \\
& =\left|\left[d-e_{1}, z-e_{2}\right]\right|
\end{aligned}
$$

we now have $|(q+\mathcal{N}) \cap \mathcal{B}|+|(p+\mathcal{N}) \cap \mathcal{B}| \geq 2 \rho^{2}+|D|+|E|+\left|\left[d-e_{1}, z-e_{2}\right]\right|$. 
Combining this with (20) we have

$$
\begin{aligned}
\left|(q+\mathcal{N}) \cap A_{\infty}\right|+\left|(p+\mathcal{N}) \cap A_{\infty}\right| \geq & |(q+\mathcal{N}) \cap \mathcal{B}|+|(p+\mathcal{N}) \cap \mathcal{B}| \\
& +\left|((q+\mathcal{N}) \cup(p+\mathcal{N})) \cap A_{t-1}\right| \\
\geq & 2 \rho^{2}+|D|+|E|+\left|\left[d-e_{1}, z-e_{2}\right]\right| \\
& +\theta-p_{1} q_{2}-p_{2} q_{1}
\end{aligned}
$$

We finish by finding good lower bounds on each of these terms. Technically, $|E|=$ $(2 \omega+1-\rho)\left(q_{2}+p_{2}+\rho-(2 \omega+1)\right)$, but we will simply use $E \geq(2 \omega+1-\rho)$. Also note that $p_{1} q_{2}+p_{2} q_{1}$ is largest when $p_{2}=q_{1}=\rho-1$. Finally, we have $|D|+\left|\left[d-e_{1}, z-e_{2}\right]\right|=\rho(2 \omega+1-\rho)$. Putting all this together we get

$$
\begin{aligned}
\left|(q+\mathcal{N}) \cap A_{\infty}\right|+\left|(p+\mathcal{N}) \cap A_{\infty}\right| \geq & 2 \rho^{2}+\rho(2 \omega+1-\rho)+(2 \omega+1-\rho) \\
& +\theta-(\rho-1)^{2}-1 \\
= & \theta+2 \rho \omega+2 \rho+2 \omega-1 \\
\geq & \theta+2 \rho \omega+2 \rho+\omega \\
\geq & 2 \theta
\end{aligned}
$$

Case 2. $\left.Q_{\alpha, \beta} \subset \mathcal{B}\right|_{\mathcal{N}}$ for some $\alpha, \beta \in\{-1,1\}$.

Assume without loss of generality that $\left.Q_{-1,-1} \subset \mathcal{B}\right|_{\mathcal{N}}$. Let $S_{z}$ be a square in $\left.\mathcal{B}\right|_{\mathcal{N}}$ for which $S_{z+e_{1}}$ and $S_{z+e_{2}}$ are not contained in $\left.\mathcal{B}\right|_{\mathcal{N}}$. Let $z=\left(-\rho+z_{1},-\rho+z_{2}\right)$. We may assume $1 \leq z_{1}, z_{2} \leq \rho+1$. We divide the argument into three subcases depending on $z$. If $z_{1}, z_{2} \leq \rho$, then by (19), there exists $p \in \bar{Q}_{1,1}$ adjacent to $S_{z}$. If exactly one of $z_{1}, z_{2}$ is $\rho+1$, then, by the definition of $S_{z}$ there exists a peg $p \in \bar{Q}_{1,1}$ adjacent to $S_{z}$. In both of these subcases we arrive at a contradiction by showing $\left|(p+\mathcal{N}) \cap A_{\infty}\right| \geq \theta$. When $z_{1}=z_{2}=\rho+1$ a much simpler argument (along the lines of what is given in Claim 8) finishes the job. Before considering the subcases we establish a simple fact concerning $\mathcal{B}$.

Let $A=\left[(0,-\rho),\left(z_{1}-1,-\rho+z_{2}-1\right)\right]$. For $a \in A$ let $a^{\prime}=a-\rho e_{1}+\rho e_{2}$. Since $S_{(-\rho,-\rho)}$ and $S_{z}$ are tight if $z \neq(1,1)$, equation (9) gives

$$
\forall a \in A \text { either } a \in \mathcal{B} \text { or } a^{\prime} \in \mathcal{B}
$$

when $z \neq(1,1)$.

Subcase 2.1. $z_{1} \leq \rho$ and $z_{2} \leq \rho$

By (19) there exists a peg $p$ adjacent to $S_{z}$ in $\bar{Q}_{1,1}$. Let $p=\left(p_{1}, p_{2}\right)$. Since $p$ is adjacent to $S_{z}$ we either have $p_{1}=z_{1}$ and $p_{2}<z_{2}$ or $p_{2}=z_{2}$ and $p_{1}<z_{1}$. We assume without loss of generality that $p_{1}=z_{1}$ and $p_{2}<z_{2}$. We establish a lower bound on $\left|(p+\mathcal{N}) \cap A_{\infty}\right|$ via

$$
\begin{aligned}
\left|(p+\mathcal{N}) \cap A_{\infty}\right| & \geq\left|\mathcal{N} \cap A_{\infty}\right|-|\mathcal{N} \backslash(p+\mathcal{N})| \\
& \geq|\mathcal{B}|_{\mathcal{N}}|+| A_{t-1} \cap \mathcal{N}|-| \mathcal{N} \backslash(p+\mathcal{N}) \mid
\end{aligned}
$$




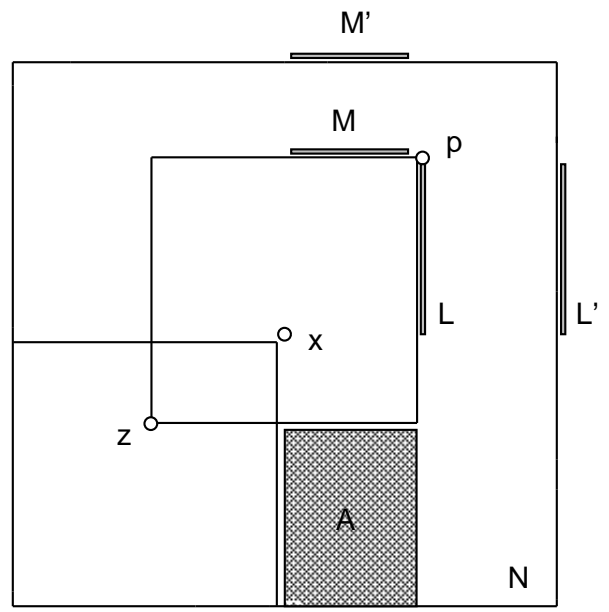

Figure 3. Subcase 2.1

By (22) we have $|\mathcal{B}|_{\mathcal{N}}|\geq| S_{z} \cup Q_{-1,-1}|+| A \mid$. A simple calculation yields $\mid S_{z} \cup$ $Q_{-1,-1}|+| A \mid=\rho^{2}+\rho z_{1}+\rho z_{2}$. Using this bound we get

$$
\begin{aligned}
\left|(p+\mathcal{N}) \cap A_{\infty}\right| \geq & \rho^{2}+\rho z_{1}+\rho z_{2}+\theta-|\mathcal{N} \backslash(p+\mathcal{N})| \\
= & \rho^{2}+\rho z_{1}+\rho z_{2}+\theta-\left((2 \rho+1) p_{1}+(2 \rho+1) p_{2}-p_{1} p_{2}\right) \\
= & \rho^{2}+\rho z_{1}+\rho z_{2}+\theta \\
& -\left((2 \rho+1) z_{1}+(2 \rho+1) z_{2}-z_{1} z_{2}\right. \\
& \left.\quad+\left(p_{2}-z_{2}\right)\left(2 \rho+1-z_{1}\right)\right) \\
= & \theta+\rho^{2}-\rho z_{1}-\rho z_{2}+z_{1} z_{2} \\
& -z_{1}-z_{2}+\left(z_{2}-p_{2}\right)\left(2 \rho+1-z_{1}\right) \\
= & \theta+\left(\rho-z_{1}\right)\left(\rho-z_{2}\right) \\
& -z_{1}-z_{2}+\left(z_{2}-p_{2}\right)\left(2 \rho+1-z_{1}\right) \\
\geq & \theta-z_{1}-z_{2}+\left(z_{2}-p_{2}\right)\left(2 \rho+1-z_{1}\right) .
\end{aligned}
$$

We are done if $z_{2} \geq p_{2}+2$ or if $2 z_{1}+z_{2} \leq 2 \rho+1$.

If neither of these conditions holds we resort to (18) in order to add another term to the lower bound. We are now restricting our attention to the situation $p_{1}=z_{1}$, $p_{2}=z_{2}-1$, and $2 z_{1}+z_{2}>2 \rho+1$. Consider the sets $L:=\left[\left(z_{1}, 0\right),\left(z_{1}, z_{2}-2\right)\right]$ and $M:=\left[\left(0, z_{2}\right),\left(z_{1}-2, z_{2}\right)\right]$. We may assume that no element of $L \cup M$ is an element of $\mathbf{Z}^{2} \backslash A_{\infty}$ (if an element $q$ of $L \cup M$ is not in $A_{\infty}$, then we can let $p$ be $q$ and we are done). We also have either $z_{1}>\rho / 2+1$ or $z_{2}>\rho / 2+1$ (this follows from $\left.2 z_{1}+z_{2}>2 \rho+1\right)$. For $a=\left(z_{1}, a_{2}\right) \in L$ let $a^{\prime}=\left(\rho+1, a_{2}\right)$ and for $a=\left(a_{1}, z_{2}\right) \in M$ let $a^{\prime}=\left(a_{1}, \rho+1\right)$. A picture of this situation is given in Figure 3 .

Now, suppose $z_{1}>\rho / 2+1$ and $a \in L$. If $a \in A_{t-1}$, then (18) implies that $w \in A_{\infty}$ when $\|w-a\|_{1} \leq \rho^{2} /(2 \rho+1)$. Now, $\left\|a-a^{\prime}\right\|_{1}=\rho+1-z_{1} \leq \rho / 2-1 / 2 \leq \rho^{2} /(2 \rho+1)$. So, either $a \in A_{\infty} \backslash A_{t-1}$ or $a^{\prime} \in A_{\infty}$. Note that if $a \in A_{\infty} \backslash A_{t-1}$, then it is not counted in (23) - the only elements of $A_{\infty} \backslash A_{t-1}$ counted in (23) are those in $\mathcal{B}$. Furthermore, if $a^{\prime} \in A_{\infty}$, then it is not counted in (23) because (23) only counts elements of $A_{\infty}$ in $\mathcal{N}$. Since both $a$ and $a^{\prime}$ are in $p+\mathcal{N}$ we can add $|L|=z_{2}-1$ to the above bound and we get a contradiction. Similarly, if $z_{2}>\rho / 2+1$ and $a \in M$, 


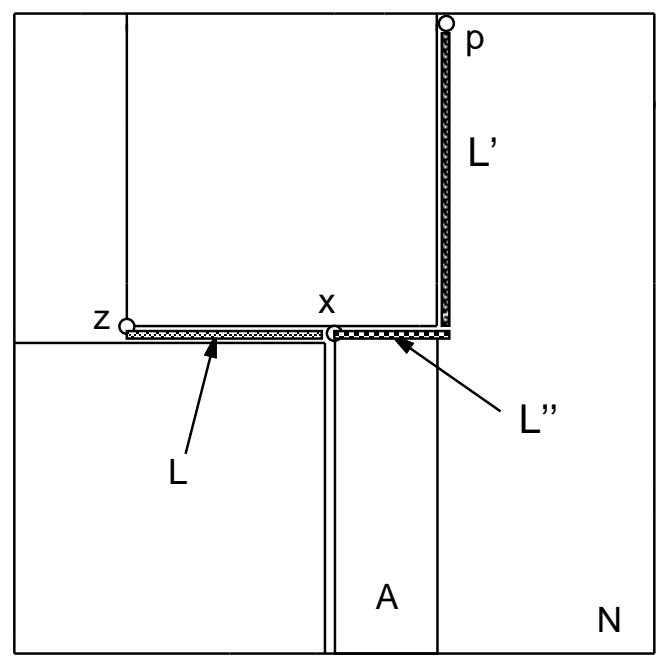

Figure 4. Subcase 2.2

then (18) implies that either $a \in A_{\infty} \backslash A-t-1$ or $a^{\prime} \in A_{\infty}$. In this case we can add $z_{1}-1$ to the above lower bound and again we have a contradiction.

Subcase 2.2. Exactly one of $z_{1}, z_{2}$ is $\rho+1$.

We assume without loss of generality that $z_{2}=\rho+1$ and $z_{1}<\rho+1$. We begin, as we did in Subcase 2.1, with calculating a lower bound on $\left|(p+\mathcal{N}) \cap A_{\infty}\right|$. Note that we must now have $p_{1}=z_{1}$ and $p_{2}<z_{2}=\rho+1$. Let $L=\left[\left(-\rho+z_{1}, 0\right),(-1,0)\right]$. Clearly, (7) implies $\left.L \subset \mathcal{B}\right|_{\mathcal{N}}$. We use this observation and (22) to get our lower bound:

$$
\begin{aligned}
\left|(p+\mathcal{N}) \cap A_{\infty}\right| \geq & \left|\mathcal{N} \cap A_{\infty}\right|-|\mathcal{N} \backslash(p+\mathcal{N})| \\
\geq & |\mathcal{B}|_{\mathcal{N}}|+| A_{t-1} \cap \mathcal{N}|-| \mathcal{N} \backslash(p+\mathcal{N}) \mid \\
\geq & \left|S_{z}\right|+\left|Q_{-1,-1}\right|+|L|+|A|+\theta \\
& -\left((2 \rho+1) p_{2}+(2 \rho+1) p_{1}-p_{1} p_{2}\right) \\
= & 2 \rho^{2}+\rho-z_{1}+z_{1}(\rho+1)+\theta \\
& -(2 \rho+1)(\rho+1)-(2 \rho+1) z_{1}+z_{1}(\rho+1) \\
& +\left(\rho+1-p_{2}\right)\left(2 \rho+1-z_{1}\right) \\
= & \theta-2 \rho-1+\left(\rho+1-p_{2}\right)\left(2 \rho+1-z_{1}\right) .
\end{aligned}
$$

We are done if $p_{2}<\rho$.

If $p_{2}=\rho$, then we may assume that the set $L^{\prime}:=\left\{\left(z_{1}, k\right): 1 \leq k<\rho\right\}$ is contained in $A_{\infty}$ (if not replace $p$ by an element of $L^{\prime}$ not in $A_{\infty}$ ). We will show that $L^{\prime} \subset \mathcal{B}$ (Figure 4 may be useful in visualizing this proof). Since $L^{\prime}$ has not been counted in the bound $|\mathcal{B}|_{\mathcal{N}}|\leq| S_{z}|+| Q_{-1,-1}|+| L|+| A \mid$ that we use in (24), if we show $L^{\prime} \subset \mathcal{B}$ then we can add $\rho$ to the bound we get in (24) and we are done. 


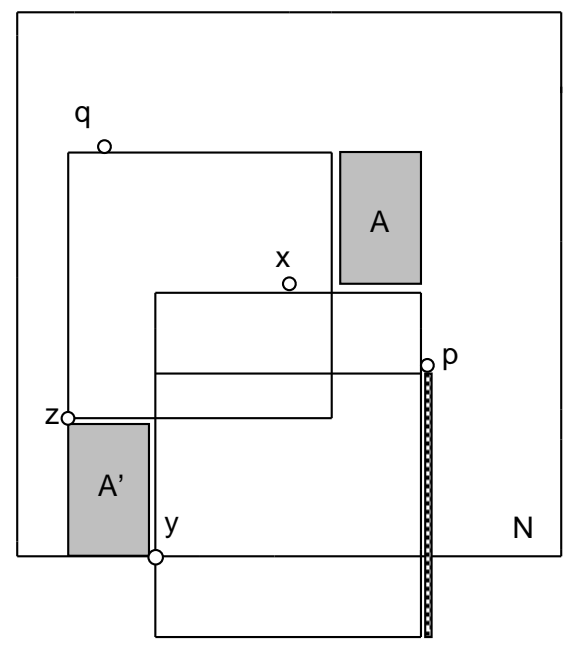

Figure 5. Case 3

Consider an arbitrary element $w=(j, 0)$ of the set $L^{\prime \prime}:=\left\{(j, 0): 1 \leq j \leq z_{1}\right\}$. The neighborhood of $w$ contains most of $\mathcal{N}$ :

$$
\begin{aligned}
\left|(w+\mathcal{N}) \cap A_{\infty}\right| & \geq|\mathcal{B}|_{\mathcal{N}}|+| A_{t-1} \cap \mathcal{N}|-| \mathcal{N} \backslash(w+\mathcal{N}) \mid \\
& \geq\left(2 \rho^{2}+(\rho+1) z_{1}\right)+\theta-(2 \rho+1) j \\
& \geq \theta+2 \rho^{2}+(\rho+1) z_{1}-(2 \rho+1) z_{1} \\
& \geq \theta .
\end{aligned}
$$

Thus, $L^{\prime} \cup L^{\prime \prime} \subset A_{\infty}$, and it follows that $L^{\prime} \cup L^{\prime \prime} \subset \mathcal{B}$.

Subcase 2.3. $z_{1}, z_{2}=\rho+1$.

If either $\bar{Q}_{1,-1} \subset \mathcal{B}$ or $\bar{Q}_{-1,1} \subset \mathcal{B}$ then, for any peg $p \in \mathcal{N}, p$ sees all of $A_{t-1} \cap \mathcal{N}$ and we have $\left|(p+\mathcal{N}) \cap A_{\infty}\right| \geq\left|A_{t-1} \cap \mathcal{N}\right| \geq \theta$. Suppose $\bar{Q}_{1,-1}, \bar{Q}_{-1,1} \not \subset \mathcal{B}$. This implies that there exist corner pegs $p \in \bar{Q}_{1,-1}$ and $q \in \bar{Q}_{-1,1}$. Since any corner peg has an intersection with $\mathcal{B}$ of cardinality at least $\rho^{2}+2 \rho$ and $A_{t-1} \cap \mathcal{N} \subset$ $\bar{Q}_{-1,1} \cup \bar{Q}_{1,-1} \subset(p+\mathcal{N}) \cup(q+\mathcal{N})$ we have

$$
\left|(p+\mathcal{N}) \cap A_{\infty}\right|+\left|(q+\mathcal{N}) \cap A_{\infty}\right| \geq 2\left(\rho^{2}+2 \rho\right)+\theta>2 \theta
$$

and we have a contradiction.

Case 3. There exists an outside corner peg p.

We assume without loss of generality that $p \in Q_{1,-1}$ with $p-\left.e_{1} \in \mathcal{B}\right|_{\mathcal{N}}$ and $p-e_{2} \in \mathcal{B}$ (it is easy to see that any outside corner peg is in a position isomorphic to this). Let $p=\left(p_{1},-p_{2}\right)$. Letting $y=\left(p_{1}-\rho,-\rho\right)$ we have $\left.S_{y} \subset \mathcal{B}\right|_{\mathcal{N}}$. Let $S_{z:=\left(-z_{1},-z_{2}\right)}$ be a square in $\left.\mathcal{B}\right|_{\mathcal{N}}$ such that $S_{z-e_{1}},\left.S_{z+e_{2}} \notin \mathcal{B}\right|_{\mathcal{N}}$. We have $\rho-p_{1} \leq$ $z_{1} \leq \rho$ (the first part of this inequality follows from (11)) and $-1 \leq z_{2} \leq \rho$. If $z_{1}=\rho$ and $z_{2}=-1$, then $Q_{-1,1} \subset \mathcal{B}$ which, by assumption, is not the case; we don't have both $z_{1}=\rho$ and $z_{2}=-1$. It follows that $S_{z}$ is bounded by some peg $q \in \mathcal{N}$. By (19) we have $q \in \bar{Q}_{-1,1}$. Let $q=\left(-q_{1}, q_{2}\right)$. For a picture of this situation, see Figure 5. 
Let $\mathcal{B}^{\prime}=S_{z} \cup S_{y} \cup S_{\mathcal{B}, p} \cup\left[p-e_{1}, p-\rho e_{1}\right] \cup R_{z, y}^{c}$. Note that $R_{z, y}^{c}$ is empty unless $z_{2}=-1$ in which case $R_{z, y}^{c}$ is the 'gap' between $S_{z}$ and $S_{y}$. Since, by (7), $R_{z, y}^{c} \subset \mathcal{B}$ we have $\mathcal{B}^{\prime} \subset \mathcal{B}$. We count as follows:

$$
\begin{aligned}
\left|(p+\mathcal{N}) \cap A_{\infty}\right|+\left|(q+\mathcal{N}) \cap A_{\infty}\right| \geq \mid & \left|(p+\mathcal{N}) \cap\left(A_{\infty} \backslash \mathcal{B}^{\prime}\right)\right| \\
& +\left|(p+\mathcal{N}) \cap \mathcal{B}^{\prime}\right| \\
& +\left|(q+\mathcal{N}) \cap\left(A_{\infty} \backslash \mathcal{B}^{\prime}\right)\right| \\
& +\left|(q+\mathcal{N}) \cap \mathcal{B}^{\prime}\right| \\
\geq & \left|(p+\mathcal{N}) \cap\left(A_{\infty} \backslash \mathcal{B}^{\prime}\right) \cap \mathcal{N}\right| \\
& +\left|(q+\mathcal{N}) \cap\left(A_{\infty} \backslash \mathcal{B}^{\prime}\right) \cap \mathcal{N}\right| \\
& +\left|(p+\mathcal{N}) \cap \mathcal{B}^{\prime}\right|+\left|(q+\mathcal{N}) \cap \mathcal{B}^{\prime}\right| \\
\geq & \left|A_{t-1} \cap \mathcal{N}\right|+|\mathcal{B}| \mathcal{N} \backslash \mathcal{B}^{\prime} \mid \\
& -|\mathcal{N} \backslash((p+\mathcal{N}) \cup(q+\mathcal{N}))| \\
& +\left|(p+\mathcal{N}) \cap \mathcal{B}^{\prime}\right|+\left|(q+\mathcal{N}) \cap \mathcal{B}^{\prime}\right| \\
\geq & \theta+|\mathcal{B}| \mathcal{N} \backslash \mathcal{B}^{\prime} \mid-p_{1} q_{2}-q_{1} p_{2} \\
& +\left|(p+\mathcal{N}) \cap \mathcal{B}^{\prime}\right|+\left|(q+\mathcal{N}) \cap \mathcal{B}^{\prime}\right| .
\end{aligned}
$$

We proceed by finding bounds on each of these terms. For ease of notation let $\alpha=z_{1}+p_{1}-\rho$ and $\beta=\rho-z_{2}$.

Let $A=\left[\left(-z_{1}+\rho, 0\right),\left(p_{1}-1, \beta-1\right)\right]$, and for $a \in A$ let $a^{\prime}=a-\rho e_{1}-\rho e_{2}$. Clearly, $A \cap \mathcal{B}^{\prime}=\emptyset$. Since $S_{y}$ and $S_{z}$ are tight, (9) implies that for all $a \in A$ either $a \in \mathcal{B}$ or $a^{\prime} \in \mathcal{B}$. Thus we have $|\mathcal{B}|_{\mathcal{N}} \backslash \mathcal{B}^{\prime}|\geq| A \mid=\alpha \beta$.

Either $q_{2}=\beta$ and $q_{1}<z_{1}+1$ or $q_{2}<\beta$ and $q_{1}=z_{1}+1$. If $q_{2}=\beta$ and $q_{1}<z_{1}+1$, we have $p_{1} q_{2}+q_{1} p_{2} \leq \beta p_{1}+\left(z_{1}+1\right) p_{2}-p_{2}$. On the other hand, if $q_{2}<\beta$ and $q_{1}=z_{1}+1$, we have $p_{1} q_{2}+q_{1} p_{2} \leq \beta p_{1}+\left(z_{1}+1\right) p_{2}-p_{1}$. For now we write $p_{1} q_{2}+p_{2} q_{1} \leq \beta p_{1}+\left(z_{1}+1\right) p_{2}-\min \left\{p_{1}, p_{2}\right\}$.

We will simply use $\rho^{2}$ as a lower bound on $\left|(q+\mathcal{N}) \cap \mathcal{B}^{\prime}\right|$. We determine the exact value of $\left|(p+\mathcal{N}) \cap \mathcal{B}^{\prime}\right|$. For this counting we consider the cases $p_{2} \leq z_{2}$ and $p_{2}>z_{2}$ separately .

If $p_{2} \leq z_{2}$, then

$$
\begin{aligned}
\left|(p+\mathcal{N}) \cap \mathcal{B}^{\prime}\right|= & \left|(p+\mathcal{N}) \cap S_{\mathcal{B}, p}\right|+\left|(p+\mathcal{N}) \cap\left(S_{y} \backslash S_{\mathcal{B}, p}\right)\right| \\
& +\left|(p+\mathcal{N}) \cap\left(S_{z} \backslash S_{y}\right)\right|+\left|(p+\mathcal{N}) \cap\left[p-e_{1}, p-\rho e_{1}\right]\right| \\
= & \rho^{2}+p_{2} \rho+\beta(\rho-\alpha)+\rho .
\end{aligned}
$$

Putting this together with the bounds we obtained above we get

$$
\begin{aligned}
\left|(p+\mathcal{N}) \cap A_{\infty}\right|+\left|(q+\mathcal{N}) \cap A_{\infty}\right| \geq \quad & +\alpha \beta-\beta p_{1}-\left(z_{1}+1\right) p_{2} \\
& +\min \left\{p_{1}, p_{2}\right\}+\rho^{2}+\rho^{2}+p_{2} \rho \\
& +\beta(\rho-\alpha)+\rho \\
\geq & 2 \theta-\beta p_{1}-z_{1} p_{2}+p_{2} \rho+\beta \rho-p_{2} \\
& +\min \left\{p_{1}, p_{2}\right\} \\
= & 2 \theta+\beta\left(\rho-p_{1}\right)+p_{2}\left(\rho-z_{1}\right)-p_{2} \\
& +\min \left\{p_{1}, p_{2}\right\} .
\end{aligned}
$$

We are done if $z_{1}<\rho$. If $z_{1}=\rho$, then $q$ is on the top of $S_{z}$ (i.e. $q_{1}<z_{1}+1$ and $\left.q_{2}=\beta\right)$. In this case, we can replace $\min \left\{p_{1}, p_{2}\right\}$ with $p_{2}$ and we're done. 
If, on the other hand, $p_{2}>z_{2}$, then

$$
\begin{aligned}
\left|(p+\mathcal{N}) \cap \mathcal{B}^{\prime}\right|= & \left|(p+\mathcal{N}) \cap S_{\mathcal{B}, p}\right|+\left|(p+\mathcal{N}) \cap\left(S_{y} \backslash S_{\mathcal{B}, p}\right)\right| \\
& +\left|(p+\mathcal{N}) \cap\left(S_{z} \backslash S_{y}\right)\right|+\left|(p+\mathcal{N}) \cap\left[p-e_{1}, p-\rho e_{1}\right]\right| \\
= & \rho^{2}+p_{2} \rho+\left(\rho+1-p_{2}\right)(\rho-\alpha)+\rho .
\end{aligned}
$$

Adding this term to the above bounds we get

$$
\begin{array}{rl}
\left|(p+\mathcal{N}) \cap A_{\infty}\right|+\left|(q+\mathcal{N}) \cap A_{\infty}\right| \geq & \theta+\alpha \beta-\beta p_{1}-\left(z_{1}+1\right) p_{2} \\
& +\min \left\{p_{1}, p_{2}\right\}+\rho^{2}+\rho^{2}+p_{2} \rho \\
& +\left(\rho+1-p_{2}\right)(\rho-\alpha)+\rho \\
\geq 2 & 2 \theta+\alpha \beta-\beta p_{1}-z_{1} p_{2}+\rho^{2}-\alpha \rho \\
& +\alpha p_{2}+\rho-\alpha-p_{2}+\min \left\{p_{1}, p_{2}\right\} \\
= & 2 \theta+\rho\left(\rho+\alpha-p_{1}-z_{1}\right) \\
& +\left(\rho-p_{2}\right)\left(z_{1}-\alpha\right) \\
& +(\rho-\beta)\left(p_{1}-\alpha\right) \\
& +\rho-\alpha-p_{2}+\min \left\{p_{1}, p_{2}\right\} \\
\geq 2 \theta+\left(\rho-p_{2}\right)\left(z_{1}-\alpha\right) & +(\rho-\beta)\left(p_{1}-\alpha\right) \\
\geq 2 &
\end{array}
$$

Note that we use the definition of $\alpha$ heavily in these inequalities. For example, $\alpha=p_{1}+z_{1}-\rho$ implies $\alpha \leq p_{1}$ and $\alpha \leq z_{1}$. It follows from $\alpha \leq p_{1}$ that $\rho-\alpha-$ $p_{2}+\min \left\{p_{1}, p_{2}\right\} \geq \rho-p_{1}-p_{2}+\min \left\{p_{1}, p_{2}\right\} \geq 0$.

Case 4. $\left.\mathcal{B}\right|_{\mathcal{N}} \cap \partial \mathcal{N}$ contains either a rectangle of the form $\left[w, w+2 \omega e_{1}\right]$ or a rectangle of the form $\left[w, w+2 \omega e_{2}\right]$.

Cases 4 and 5 are different from the previous cases in that there is no obvious set of pegs whose neighborhoods cover most of $\mathcal{N}$. So most of the work here will be in finding 'good' pegs. We assume without loss of generality that

$$
\left.\left[\left(w_{1},-\rho\right),\left(w_{1}+2 \omega,-\rho\right)\right] \subset \mathcal{B}\right|_{\mathcal{N}} \cap \partial \mathcal{N}
$$

(so $w=\left(w_{1},-\rho\right)$ ). Let $\left.S_{z} \subset \mathcal{B}\right|_{\mathcal{N}}$ be a square for which $S_{z+e_{1}}, S_{z+e_{2}} \not \subset \mathcal{B}$. Since, by assumption, $Q_{1,1} \not \subset \mathcal{B}, S_{z}$ is bounded by some peg $q$ in $\mathcal{N}$. By (19) we can choose $q \in \bar{Q}_{1,1}$. Similarly, there exists a peg $p$ of $\mathcal{B}$ in $\bar{Q}_{-1,1}$.

In order to both handle an important subcase and indicate how the other subcases will be dealt with, suppose $p$ and $q$ are corner pegs. This gives $\mid(p+\mathcal{N}) \cap$ $\mathcal{B}||,(q+\mathcal{N}) \cap \mathcal{B} \mid \geq \rho^{2}+2 \rho$ (if $p+\alpha e_{1}, p+\beta e_{2} \in \mathcal{B}$, then

$$
\left.S_{\mathcal{B}, p} \cup\left(S_{\mathcal{B}, p}-\alpha e_{1}\right) \cup\left(S_{\mathcal{B}, p}-\beta e_{2}\right) \subset(p+\mathcal{N}) \cap \mathcal{B}\right) .
$$

The set $(p+\mathcal{N}) \cup(q+\mathcal{N})$ covers $\bar{Q}_{1,-1} \cup \bar{Q}_{1,1}$. Since there are at most $\rho(2 \rho-2 \omega)$ elements of $\mathcal{N} \backslash \mathcal{B} \backslash \bar{Q}_{1,-1} \backslash \bar{Q}_{1,1}$ we have 


$$
\begin{aligned}
\left|(p+\mathcal{N}) \cap A_{\infty}\right|+\left|(q+\mathcal{N}) \cap A_{\infty}\right| \geq & \left|((p+\mathcal{N}) \cup(q+\mathcal{N})) \cap\left(A_{t-1} \cap \mathcal{N}\right)\right| \\
& +|(p+\mathcal{N}) \cap \mathcal{B}|+|(q+\mathcal{N}) \cap \mathcal{B}| \\
\geq & \left|A_{t-1} \cap \mathcal{N}\right| \\
& -|\mathcal{N} \backslash \mathcal{B} \backslash((p+\mathcal{N}) \cup(q+\mathcal{N}))| \\
& +|(p+\mathcal{N}) \cap \mathcal{B}|+|(q+\mathcal{N}) \cap \mathcal{B}| \\
\geq & \theta-\rho(2 \rho-2 \omega)+2\left(\rho^{2}+2 \rho\right) \\
= & \theta+2 \rho \omega+4 \rho \\
\geq & \theta+2 \rho \omega+2 \rho+\omega \\
\geq & 2 \theta .
\end{aligned}
$$

So we're done when we can find a corner peg in $\bar{Q}_{-1,1}$ and a corner peg in $\bar{Q}_{1,1}$. When we don't have these two corner pegs we will argue that we can find $p \in \bar{Q}_{-1,1}$ and $q \in \bar{Q}_{1,1}$ such that

$$
|(p+\mathcal{N}) \cap \mathcal{B}|+|(q+\mathcal{N}) \cap \mathcal{B}| \geq 2 \rho^{2}+2 \rho+\omega .
$$

Appealing to calculation (25) it is clear that (26) implies $\left|(p+\mathcal{N}) \cap A_{\infty}\right|+\mid(q+$ $\mathcal{N}) \cap A_{\infty} \mid \geq 2 \theta$. Before considering more cases we establish the following fact.

Claim 12. If there exists $z=\left(z_{1}, z_{2}\right)$ with $z_{2} \leq 0, S_{z}, S_{z+e_{1}},\left.S_{z+2 e_{2}} S_{z+3 e_{1}} \subset \mathcal{B}\right|_{\mathcal{N}}$ and $S_{z+e_{2}}, S_{z+e_{1}+e_{2}}, S_{z+2 e_{1}+e_{2}},\left.S_{z+3 e_{1}+e_{2}} \not \subset \mathcal{B}\right|_{\mathcal{N}}$, then there exist pegs $p \in \bar{Q}_{-1,1}$ and $q \in \bar{Q}_{1,1}$ which satisfy (26).

Proof. Let $L=\left[\left(z_{1}+2, z_{2}+\rho\right),\left(z_{1}+\rho, z_{2}+\rho\right)\right], D=\left\{\left(z_{1}, z_{2}+\rho\right),\left(z_{1}+1, z_{2}+\rho\right)\right\}, E=$ $\left\{\left(z_{1}+\rho+1, z_{2}+\rho\right),\left(z_{1}+\rho+2, z_{2}+\rho\right)\right\}$, and $F=\left\{\left(z_{1}+1, z_{2}+\rho\right),\left(z_{1}+\rho+1, z_{2}+\rho\right)\right\}$. If there is a peg in $L$, then we are done (for $p$ and $q$ we choose a peg in $L$ and any peg in the other quadrant). If there is no peg in $L$, then there must be at least one peg in each of $D, E$, and $F$. Let $p$ be a peg in $D$ and $q$ be a peg in $E$ so that either $p$ or $q$ is in $F$. Since $p \in D$ we have $p \in \bar{Q}_{-1,1}$ and $|(p+\mathcal{N}) \cap \mathcal{B}| \geq \rho^{2}+\rho$. Since $q \in E$ we have $q \in \bar{Q}_{1,1}$ and $|(q+\mathcal{N}) \cap \mathcal{B}| \geq \rho^{2}+\rho$. Since $r \in F$ implies $|(r+\mathcal{N}) \cap \mathcal{B}| \geq \rho^{2}+2 \rho$ (and since $\left.\rho \geq \omega\right)$ this choice of $p$ and $q$ gives (26).

Subcase 4.1. There are no corner pegs in $\bar{Q}_{-1,1} \cup \bar{Q}_{1,1}$.

In this case $R:=\mathcal{B} \cap\left(\bar{Q}_{1,1} \cap \bar{Q}_{-1,1}\right)$ is a rectangle. Let $R=\left[\left(y_{1}, 0\right),\left(z_{1}, z_{2}\right)\right]$ where $y_{1}<z_{1}$. Note that we must have $y_{1} \leq w_{1}$ and $z_{1} \geq w_{1}+2 \omega$. We consider subcases.

Subcase 4.1.1. $z_{2}=\rho$.

In this case $\left[w, w+2 \rho e_{2}+2 \omega e_{1}\right] \subset \mathcal{B}$. Instead of looking for $p \in \bar{Q}_{-1,1}$ with $|(p+\mathcal{N}) \cap \mathcal{B}|$ large we look for $p \in \bar{Q}_{-1,1} \cup \bar{Q}_{-1,-1}$ with $|(p+\mathcal{N}) \cap \mathcal{B}| \geq \rho^{2}+2 \rho$. If there is a corner peg in $\bar{Q}_{-1,-1}$ we let $p$ be this corner peg. If no such corner peg exists, then $\mathcal{B} \cap\left(\bar{Q}_{-1,1} \cup \bar{Q}_{-1,-1}\right)=\left[\left(y_{1},-\rho\right),(0, \rho)\right]$, and we let $p$ be any peg in $\left[\left(y_{1}-1,-\rho+1\right),\left(y_{1}-1, \rho-1\right)\right]$ (clearly, at least one such peg must exist). Similarly, there exists $q \in \bar{Q}_{1,1} \cup \bar{Q}_{1,-1}$ with $|(q+\mathcal{N}) \cap \mathcal{B}| \geq \rho^{2}+2 \rho$. Since $\left[w, w+2 \rho e_{2}+2 \omega e_{1}\right] \subset$ $\mathcal{B}$ there are at most $\rho(2 \rho-2 \omega)$ elements of $\mathcal{N} \backslash \mathcal{B} \backslash(p+\mathcal{N}) \backslash(q+\mathcal{N})$, and following (25) we arrive at a contradiction.

Throughout the following two subcases we assume $z_{2}<\rho$.

Subcase 4.1.2. $y_{1}=w_{1}$ and $z_{1}=w_{1}+2 \omega$. 
In this case $\left.\mathcal{B}\right|_{\mathcal{N}}=\left[w,\left(z_{1}, z_{2}\right)\right]$. From this observation it follows that for any peg $r \in \bar{Q}_{-1,1} \cup \bar{Q}_{1,1}$ we have $|(r+\mathcal{N}) \cap \mathcal{B}| \geq \rho^{2}+\rho$. So to achieve (26) it suffices to find a peg $s \in \bar{Q}_{-1,1} \cup \bar{Q}_{1,1}$ for which $|(s+\mathcal{N}) \cap \mathcal{B}| \geq \rho^{2}+2 \rho$. Such a peg exists since there must be a peg in $\left[\left(y_{1}+1, z_{2}+1\right),\left(z_{1}-1, z_{2}+1\right)\right]$ (here we are using $\omega \geq \rho / 2+1 / 2)$.

Subcase 4.1.3. $y_{1}<w_{1}$ or $z_{1}>w_{1}+2 \omega$.

Here we can apply Claim 12 (take $z$ in the statement of Claim 12 to be $\left(y_{1}, z_{2}-\right.$ $\rho+1))$.

Subcase 4.2. There exists a corner peg in $\bar{Q}_{-1,1}$ and there are no corner pegs in $\bar{Q}_{1,1}$.

Let $p$ be a corner peg in $\bar{Q}_{-1,1}$ and $q$ be a peg in $\bar{Q}_{1,1}$. Any of the following conditions imply (26):

(a) $\left|\left\{x \in \mathcal{B}:\|x-p\|_{\infty}=1\right\}\right| \geq 4$.

(b) There is a corner peg in $\left\{p \pm e_{1} \pm e_{2}\right\}$.

(c) $|(q+\mathcal{N}) \cap \mathcal{B}| \geq \rho^{2}+\rho-1$.

Note that for $(b)$ and $(c)$ we are using $\omega<\rho$ (this follows from the fact that $\mathcal{B}$ does not contain an open quadrant).

Let $S_{y}$ where $y=\left(y_{1}, y_{2}\right)$ be a square in $\left.\mathcal{B}\right|_{\mathcal{N}}$ which satisfies $S_{y+e_{1}},\left.S_{y+e_{2}} \notin \mathcal{B}\right|_{\mathcal{N}}$. By (12), $y_{1} \geq w_{1}+2 \omega-\rho+1$. Let $q$ be any peg in $\bar{Q}_{1,1}$ adjacent to $S_{y}$. We now consider subcases.

Subcase 4.2.1. $q-e_{2} \in S_{y}$.

If $r:=\left(y_{1}-1, y_{2}+\rho-1\right)$ is an element of $\mathcal{B}$, then $S_{y}-e_{1}$ is a subset of $\mathcal{B}$ and $q$ satisfies $(c)$. If $r$ is a corner peg, then $S_{y}-e_{1}-e_{2} \subset \mathcal{B}$ and $q$ satisfies $(c)$. If $r$ is neither a corner peg nor an element of $\mathcal{B}$, then there exists a corner peg $p \in \bar{Q}_{-1,1}$ which satisfies $(a)$ (choose the corner peg in $\left.\left(S_{y}-e_{1}\right) \backslash S_{y}\right)$.

We must eliminate the following two situations before considering the case $q-e_{1} \in$ $S_{y}$.

Subcase 4.2.2. $y_{2}=1$.

Since there are no corner pegs in $\bar{Q}_{1,1}$ we must have $S_{y}-e_{2} \subset \mathcal{B}$. Since $q+\mathcal{N}$ contains $S_{y} \cup\left(S_{y}-e_{2}\right), q$ satisfies $(c)$.

Subcase 4.2.3. $y_{2}<1$ and there exists $u=\left(u_{1}, u_{2}\right) \in \mathcal{B}$ with $u_{2} \geq y_{2}+\rho$.

By (12) there is a corner peg $p$ in $S_{y}+e_{2}$. Since there are no corner pegs in $\bar{Q}_{1,1}$, $p$ must lie in $Q_{-1,1}$. It follows (due the fact that $w_{1}+2 \omega \geq 2$ ) that $p$ satisfies $(a)$ (i.e. $p \in S_{y}-e_{1}+e_{2}$ ).

Subcase 4.2.4. $q-e_{1} \in S_{y}, y_{2}<1$, and $\max \left\{u_{2}: \exists u_{1}\right.$ such that $\left.\left(u_{1}, u_{2}\right) \in \mathcal{B}\right\}=$ $y_{2}+\rho-1$.

Let $z_{1}=\min \left\{i: S_{\left(i, y_{2}\right)} \subset \mathcal{B}\right\}$ and let $z=\left(z_{1}, y_{2}\right)$. If $y_{1}-z_{1} \geq 3$, then we have (26) by Claim 12. If $z_{1}-w_{1} \geq 2$, then there exists a peg in $\bar{Q}_{-1,1}$ satisfying either $(a)$ or $(b)$ (to find $p$ consider the corner pegs in $\left.\left(S_{z}-2 e_{1}\right) \backslash S_{z}\right)$. So we are done unless $y_{1}-w_{1} \leq 3$. But, when $y_{1}-w_{1} \leq 3$ we have $w_{1}+\rho+2 \leq q_{1} \leq w_{1}+\rho+3$ while, by the definition of $\omega, 2 \omega+1 \geq \rho+2$. So $\left|(q+\mathcal{N}) \cap\left(\left[w, w+(\rho+1) e_{1}+(\rho-1) e_{2}\right] \backslash S_{y}\right)\right| \geq \rho-1$ and we have $(c)$. 
Case 5. $c(\mathcal{B}) \subset \mathcal{N}$.

In this case we immediately get a contradiction for many values of $\rho$ and $\theta$ by the following observation: for the center of $\mathcal{B}$ to be contained in $\mathcal{N}$ and the origin to be one of the first elements of $\mathcal{B}$ to crystallize we must have

$$
|c(\mathcal{B})|+\theta \leq|\mathcal{N}| .
$$

It is easy to check that when $\rho=2$ and $\theta>4$ (recall that we assume $\theta>\rho^{2}$ ) equation (27) is never satisfied. It is also easy to check that Theorem 1 follows directly from Lemma 2 when $\rho=1$. So we henceforth assume $\rho \geq 3$. For an arbitrary $\rho$ we can use (27) to eliminate many of the larger values of $\theta$ from consideration (we don't use this fact in what follows).

As in Case 4 the main problem here is to identify a set of pegs whose neighborhoods cover most of $\mathcal{N}$. Since our main method for finding these pegs fails when $\theta$ is smaller than $4 \rho^{2} / 3+2 \rho$, we consider two subcases.

Subcase 5.1. $\theta>4 \rho^{2} / 3+2 \rho$.

As a first step in identifying a set of pegs whose neighborhoods cover most of $\mathcal{N}$ we establish the following claim concerning the rectangle $A:=[(-\lfloor\rho / 2\rfloor,-\lfloor\rho / 2\rfloor)$, $(\lfloor\rho / 2\rfloor,\lfloor\rho / 2\rfloor)]$. When $\rho$ is odd $A$ is $S_{(-\lfloor\rho / 2\rfloor,-\lfloor\rho / 2\rfloor)}$, and when $\rho$ is even $A$ is the $(\rho+1) \times(\rho+1)$ square centered at the origin.

Claim 13. $A \subset \mathcal{B}$.

Proof. It is enough to show $A \subset A_{\infty}$. To see that this is enough assume $A \subset A_{\infty}$ and consider a square $\left.S_{z=\left(z_{1}, z_{2}\right)} \subset \mathcal{B}\right|_{\mathcal{N}}$ such that $S_{z} \not \subset A$ and $\left|S_{z} \cap A\right|$ is maximal. Assume without loss of generality that $z_{1}, z_{2}<\lfloor\rho / 2\rfloor$. Clearly, $S_{z}+e_{1}, S_{z}+e_{2} \not \subset \mathcal{B}$. By (19) there is a peg adjacent to $S_{z}$ in $\bar{Q}_{1,1}$. However, $\left(S_{z}+e_{1}\right) \cap \bar{Q}_{1,1},\left(S_{z}+e_{2}\right) \cap$ $\bar{Q}_{1,1} \subset A \subset A_{\infty}$, and we have a contradiction.

To show $A \subset A_{\infty}$ consider $v \in A$. Clearly,

$$
\begin{aligned}
\left|(v+\mathcal{N}) \cap A_{\infty}\right| & \geq\left|\mathcal{N} \cap A_{\infty}\right|-|\mathcal{N} \backslash(v+\mathcal{N})| \\
& \geq|c(\mathcal{B})|+\left|A_{t-1} \cap \mathcal{N}\right|-|\mathcal{N} \backslash(v+\mathcal{N})| \\
& \geq(2 \omega+1)^{2}-4+\theta-|\mathcal{N} \backslash(v+\mathcal{N})| .
\end{aligned}
$$

So we can conclude that $v$ is in $A_{\infty}$ when $(2 \omega+1)^{2}-4-|\mathcal{N} \backslash(v+\mathcal{N})| \geq 0$. For elements of $A$, the quantity $|\mathcal{N} \backslash(v+\mathcal{N})|$ is at its maximum when $v=(\lfloor\rho / 2\rfloor,\lfloor\rho / 2\rfloor)$. Using $\omega \geq 2 \rho / 3$ (which follows from $\theta>4 \rho^{2} / 3+2 \rho$ ) we get

$$
\begin{aligned}
(2 \omega+1)^{2}-4-|\mathcal{N} \backslash(v+\mathcal{N})| \geq & 16 \rho^{2} / 9+8 \rho / 3-3 \\
& -2(\rho / 2)(2 \rho+1)+(\rho / 2)^{2} \\
= & \rho^{2} / 36+5 \rho / 3-3 \\
\geq & 0 .
\end{aligned}
$$

Now, for $\alpha \in\{-1,1\}$ let $h_{\alpha}$ be the largest integer for which $A+\left.\alpha h_{\alpha} e_{1} \subset \mathcal{B}\right|_{\mathcal{N}}$. Analogously, for $\beta \in\{-1,1\}$ let $v_{\beta}$ be the largest integer for which $A+\left.\beta v_{\beta} e_{2} \subset \mathcal{B}\right|_{\mathcal{N}}$. Let $A^{\prime}=A \cup\left(A+h_{1} e_{1}\right) \cup\left(A-h_{-1} e_{1}\right)$ and $A^{\prime \prime}=A \cup\left(A+v_{1} e_{2}\right) \cup\left(A-v_{-1} e_{2}\right)$. We will consider the pegs which define $A^{\prime}$ and $A^{\prime \prime}$. It turns out that the neighborhoods of these pegs cover most of $\mathcal{N}$. We divide the argument into subcases depending on the values of $h_{1}, h_{-1}, v_{1}$, and $v_{-1}$. Throughout this discussion we assume that 
$\rho$ is odd. The calculations are nearly identical when $\rho$ is even; we leave them to the reader.

Subcase 5.1.1. $h_{1}, h_{-1}<\lceil\rho / 2\rceil$.

Let $p \in\left(A+\left(h_{1}+1\right) e_{1}\right) \backslash\left(A+h_{1} e_{1}\right)$ and $q \in\left(A-\left(h_{-1}+1\right) e_{1}\right) \backslash\left(A-h_{-1} e_{1}\right)$ be pegs. We count as follows:

$$
\begin{aligned}
\left|(p+\mathcal{N}) \cap A_{\infty}\right|+\left|(q+\mathcal{N}) \cap A_{\infty}\right| \geq & \left|(p+\mathcal{N}) \cap A^{\prime}\right|+\left|(q+\mathcal{N}) \cap A^{\prime}\right| \\
& +\left|((p+\mathcal{N}) \cup(q+\mathcal{N})) \cap\left(A_{\infty} \backslash A^{\prime}\right)\right| \\
\geq & \left|(p+\mathcal{N}) \cap A^{\prime}\right|+\left|(q+\mathcal{N}) \cap A^{\prime}\right| \\
& +\left|\left(\mathcal{B} \backslash A^{\prime}\right) \cap \mathcal{N}\right|+\left|A_{t-1} \cap \mathcal{N}\right| \\
& -|\mathcal{N} \backslash((p+\mathcal{N}) \cup(q+\mathcal{N}))| .
\end{aligned}
$$

Clearly, $\left|(p+\mathcal{N}) \cap A^{\prime}\right|=\left|(q+\mathcal{N}) \cap A^{\prime}\right|=\rho^{2}$. Since $c(\mathcal{B}) \subset N$,

$$
\begin{aligned}
\left|\left(\mathcal{B} \backslash A^{\prime}\right) \cap \mathcal{N}\right| & \geq|c(\mathcal{B}) \backslash[(-\rho,-\lfloor\rho / 2\rfloor),(\rho,\lfloor\rho / 2\rfloor)]| \\
& \geq(2 \omega+1-\rho)(2 \omega+1)-4 .
\end{aligned}
$$

Finally, since $(p+\mathcal{N}) \cup(q+\mathcal{N})$ covers $[(-\rho,-\lceil\rho / 2\rceil),(\rho,\lceil\rho / 2\rceil)]$ as well as the quadrants containing $p$ and $q$, we have $|\mathcal{N} \backslash((p+\mathcal{N}) \cup(q+\mathcal{N}))| \leq(\rho / 2-1 / 2)(2 \rho+1)$. Putting all this information together we get:

$$
\begin{aligned}
\left|(p+\mathcal{N}) \cap A_{\infty}\right|+\left|(q+\mathcal{N}) \cap A_{\infty}\right| \geq & 2 \rho^{2}+\theta+(2 \omega+1-\rho)(2 \omega+1)-4 \\
& -(\rho / 2-1 / 2)(2 \rho+1) \\
\geq \quad & \theta+\rho^{2}+4 \omega^{2}-2 \rho \omega \\
& +4 \omega-\rho / 2-5 / 2 \\
\geq & \theta+(2 \omega \rho+2 \rho+\omega)+(2 \omega-\rho)^{2} \\
& +3 \omega-5 / 2 \rho-5 / 2 \\
\geq & 2 \theta+\rho^{2} / 9-\rho / 2-5 / 2 .
\end{aligned}
$$

This gives our contradiction when $\rho \geq 7$.

To handle the remaining cases $(\rho=3,5)$ we first note that we are done if we can add 2 to our lower bound on $\left|(p+\mathcal{N}) \cap A_{\infty}\right|+\left|(q+\mathcal{N}) \cap A_{\infty}\right|$. Note that we can decrease our upper bound on $|\mathcal{N} \backslash((p+\mathcal{N}) \cup(q+\mathcal{N}))|$ by at least $\rho$ if either $\left|p_{2}\right|<\rho / 2-1 / 2$ or $\left|q_{2}\right|<\rho / 2-1 / 2$. So assume we can only choose $p$ and $q$ with $\left|p_{2}\right|=\left|q_{2}\right|=\rho / 2-1 / 2$. Under this assumption there exists $p^{\prime} \in$ $\left(A+\left(h_{1}+1\right) e_{1}\right) \backslash\left(A+h_{1} e_{1}\right)$ such that $p^{\prime}$ is adjacent to $p$ and $p \in A_{\infty}$. We claim that $p^{\prime}$ is an element of $(p+\mathcal{N}) \cap A_{\infty}$ that has not been counted in the above calculation: by (18) $p^{\prime}$ is not in $A_{t-1}$, by definition $p^{\prime} \notin A^{\prime}$, and $p^{\prime}$ is not in $c(\mathcal{B}) \backslash[(-\rho,-\lfloor\rho / 2\rfloor),(\rho,\lfloor\rho / 2\rfloor)]$. Similarly, there exists $q^{\prime}$ adjacent to $q$ such that $q^{\prime} \in A_{\infty}$ and $q^{\prime}$ has not been counted in the above calculation. So we can increase our lower bound on $\left|(p+\mathcal{N}) \cap A_{\infty}\right|+\left|(q+\mathcal{N}) \cap A_{\infty}\right|$ by two and we have our contradiction.

Subcase 5.1.2. $h_{-1}, v_{-1}=\lceil\rho / 2\rceil$ and $h_{1}, v_{1}<\lceil\rho / 2\rceil$.

Let $p=\left(p_{1}, p_{2}\right)$ be a peg in $\left(A+\left(h_{1}+1\right) e_{1}\right) \backslash\left(A+h_{1} e_{1}\right)$ and let $q=\left(q_{1}, q_{2}\right)$ be a peg in $\left(A+\left(v_{1}+1\right) e_{2}\right) \backslash\left(A+v_{1} e_{2}\right)$. Assume without loss of generality that $p_{1} \leq q_{2}$. 
We show that $p+\mathcal{N}$ contains at least $\theta$ elements of $A_{\infty}$. We count as follows:

$$
\begin{aligned}
\left|(p+\mathcal{N}) \cap A_{\infty}\right| \geq & \left|(p+\mathcal{N}) \cap A^{\prime}\right|+\left|A_{t-1} \cap \mathcal{N}\right| \\
& +\left|\left(B \backslash A^{\prime}\right) \cap \mathcal{N}\right|-\left|\mathcal{N} \backslash A^{\prime} \backslash(p+\mathcal{N})\right| \\
\geq & \left|(p+\mathcal{N}) \cap A^{\prime}\right|+\left|A_{t-1} \cap \mathcal{N}\right| \\
& +\left|A^{\prime \prime} \backslash A^{\prime}\right|-\left|\mathcal{N} \backslash A^{\prime} \backslash(p+\mathcal{N})\right| .
\end{aligned}
$$

Since $\left|\mathcal{N} \backslash A^{\prime} \backslash(p+\mathcal{N})\right|=p_{1}(\rho+1)+\left|p_{2}\right|(2 \rho+1)-p_{1}\left|p_{2}\right|$ and $\left|p_{2}\right| \leq \rho / 2-1 / 2$ we have

$$
\begin{aligned}
\left|(p+\mathcal{N}) \cap A_{\infty}\right| \geq & \rho^{2}+\theta+\left(\rho(\rho / 2+1 / 2)+\left(q_{2}-\rho / 2-1 / 2\right) \rho\right) \\
& -\left(p_{1}(\rho+1)+\left|p_{2}\right|(2 \rho+1)-p_{1}\left|p_{2}\right|\right) \\
\geq & \theta+\rho^{2}+q_{2} \rho-p_{1}(\rho / 2+3 / 2)-(\rho / 2-1 / 2)(2 \rho+1) \\
\geq & \theta+\rho / 2+1 / 2 .
\end{aligned}
$$

Subcase 5.1.3. $h_{1}, h_{-1}, v_{-1}=\lceil\rho / 2\rceil$ and $v_{1}<\lceil\rho / 2\rceil$.

Let $p$ be a peg in $\left(A+\left(v_{1}+1\right) e_{2}\right) \backslash\left(A+v_{1} e_{2}\right)$. We count as follows:

$$
\begin{aligned}
\left|(p+\mathcal{N}) \cap A_{\infty}\right| \geq & \left|(p+\mathcal{N}) \cap A^{\prime \prime}\right|+\left|(p+\mathcal{N}) \cap\left(A^{\prime} \backslash A^{\prime \prime}\right)\right| \\
& +\left|(p+\mathcal{N}) \cap A_{t-1}\right|-\left|\mathcal{N} \backslash A^{\prime} \backslash A^{\prime \prime} \backslash(p+\mathcal{N})\right| .
\end{aligned}
$$

Clearly $\left|(p+\mathcal{N}) \cap A^{\prime \prime}\right|=\rho^{2}$ and $\left|(p+\mathcal{N}) \cap\left(A^{\prime} \backslash A^{\prime \prime}\right)\right| \geq \rho / 2+1 / 2$. Since $(p+\mathcal{N})$ covers $[(-\lceil\rho / 2\rceil, 0),(\lceil\rho / 2\rceil, \rho)]$ and the quadrant containing $p$, we have

$$
\begin{aligned}
\left|\mathcal{N} \backslash A^{\prime} \backslash A^{\prime \prime} \backslash(p+\mathcal{N})\right| & \leq(\rho+1)(\rho / 2+1 / 2)+(\rho / 2+1 / 2)(\rho / 2-1 / 2) \\
& =3 / 4 \rho^{2}+\rho+1 / 4 . \\
\left|(p+\mathcal{N}) \cap A_{\infty}\right| & \geq \rho^{2}+(\rho / 2+1 / 2)+\theta-3 / 4 \rho^{2}-\rho-1 / 4 \\
& =\theta+1 / 4 \rho^{2}-\rho / 2+1 / 4 \\
& >\theta .
\end{aligned}
$$

Subcase 5.1.4. $h_{1}, h_{-1}, v_{-1}, v_{1}=\lceil\rho / 2\rceil$.

In this case we have $|\mathcal{B}|_{\mathcal{N}}|\geq| A^{\prime} \cup A^{\prime \prime} \mid=3 \rho^{2}+2 \rho$ and

$$
\left|A_{\infty} \cap \mathcal{N}\right| \geq\left|A_{t-1} \cap \mathcal{N}\right|+|\mathcal{B} \cap \mathcal{N}|>4 / 3 \rho^{2}+2 \rho+3 \rho^{2}+2 \rho \geq|\mathcal{N}| .
$$

This is a contradiction.

Subcase 5.2. $\theta \leq 4 / 3 \rho^{2}+2 \rho$.

Here we modify the argument given in Claim 8. Namely, we show that if $c(\mathcal{B}) \subset$ $\mathcal{N}$ (and we assume that we are in none of the above cases), then there exists a set of pegs $\left\{p_{1,1}, p_{1,-1}, p_{-1,1}, p_{-1,-1}\right\}$ such that $p_{\alpha, \beta} \in \bar{Q}_{\alpha, \beta}$ and $\sum\left|\left(p_{\alpha, \beta}+\mathcal{N}\right) \cap \mathcal{B}\right| \geq$ $4 \rho^{2}+6 \rho$. One of these pegs sees at least $\rho^{2}+3 / 2 \rho+\theta / 4 \geq \theta$ elements of $A_{\infty}$.

Choose $p_{\alpha, \beta} \in \bar{Q}_{\alpha, \beta}$ such that $p_{\alpha, \beta}$ is a peg and

$$
\left|\left(p_{\alpha, \beta}+\mathcal{N}\right) \cap \mathcal{B}\right|=\max \left\{|(p+\mathcal{N}) \cap \mathcal{B}|: p \in \bar{Q}_{\alpha, \beta} \text { and } p \text { is a peg }\right\} .
$$

Let $P=\left\{p_{1,1}, p_{1,-1}, p_{-1,1}, p_{-1,-1}\right\}, S=\left\{p \in P:|(p+\mathcal{N}) \cap \mathcal{B}| \geq \rho^{2}+2 \rho\right\}$ and $T=\left\{p \in P:|(p+\mathcal{N}) \cap \mathcal{B}|<\rho^{2}+\rho\right\}$.

Claim 14. $|S| \geq 2$. 
Proof. Suppose $c(\mathcal{B})=C_{z}$. Let $A=\left[z-(\omega-1) e_{1}-\omega e_{2}, z+(\omega-1) e_{1}+\omega e_{2}\right]$. Let $i$ be the largest integer for which $A+\left.i e_{1} \subset \mathcal{B}\right|_{\mathcal{N}}$. Note that $\left(A+i e_{1}\right) \cap \partial \mathcal{N}=\emptyset$ since we are assuming that we are not in Case 4. If there exists $S_{y}$ such that $S_{y} \subset A+i e_{1}, S_{y}+e_{1} \subset \mathcal{B}$, and $S_{y}+e_{1} \not \subset A+i e_{1}$, then there is a corner peg in $\left(A+(i+1) e_{1}\right) \backslash\left(A+i e_{1}\right)$. If no such $S_{y}$ exists, then any peg in the central $\rho$ elements of $\left(A+(i+1) e_{1}\right) \backslash\left(A+i e_{1}\right)$ is in $S$. Thus, there is at least one element of $S$ in $\bar{Q}_{1,1} \cup \bar{Q}_{1,-1}$. Similarly, there is at least one element of $S$ in $\bar{Q}_{-1,1} \cup \bar{Q}_{-1,-1}$.

Claim 15. If $p:=p_{\alpha, \beta} \in T$, then there is a corner peg in $p+\mathcal{N}$.

Proof. Let $S_{y}$ be a square in $\left.\mathcal{B}\right|_{\mathcal{N}}$ adjacent to $p$. If $p-\alpha e_{1} \in S_{y}$, then $S_{y}-\beta e_{2}$ is contained in $p+\mathcal{N}$ and not contained in $\mathcal{B}$. There is a corner peg in $\left(S_{y}-\beta e_{2}\right) \backslash S_{y}$. Similarly, if $p-\beta e_{2} \in \mathcal{B}$, then there is a corner peg in $\left(S_{z}-\alpha e_{1}\right) \backslash S_{z}$.

We define a function $\psi: T \rightarrow S$ by

$$
\psi(p)= \begin{cases}p_{\alpha,-\beta}, & p-\alpha e_{1} \in \mathcal{B} \\ p_{-\alpha, \beta}, & p-\beta e_{2} \in \mathcal{B}\end{cases}
$$

Note that if $\psi^{-1}(s) \neq \emptyset$, then $s$ is a corner peg. We handle this case by showing that for any $s \in S$ we have $\sum_{p \in \psi^{-1}(s)}|(p+\mathcal{N}) \cap \mathcal{B}|+|(s+\mathcal{N}) \cap \mathcal{B}| \geq\left(\left|\psi^{-1}(s)\right|+1\right)\left(\rho^{2}+\rho\right)$.

Subcase 5.2.1. $\left|\psi^{-1}(s)\right|=1$.

Assume without loss of generality that $s=p_{1,-1}$ and that $\psi\left(p_{1,1}\right)=s$. Let $S_{y=\left(y_{1}, y_{2}\right)}$ be the square in $\mathcal{B}$ adjacent to $p_{1,1}$. Since there are no corner squares in $\bar{Q}_{1,1}, \max \left\{y:\left.\exists(x, y) \in \mathcal{B}\right|_{\mathcal{N}}\right\}=y_{2}+\rho-1$. Since $\left.c(\mathcal{B}) \subset \mathcal{B}\right|_{\mathcal{N}}$ there exists $\left.S_{z=\left(z_{1}, z_{2}\right)} \subset \mathcal{B}\right|_{\mathcal{N}}$ such that $z_{2} \leq y_{2}-2$. Now, if either $S_{y}-e_{1}-e_{2}$ or $S_{y}-2 e_{1}-2 e_{2}$ is not contained in $\left.\mathcal{B}\right|_{\mathcal{N}}$, then $|(s+\mathcal{N}) \cap \mathcal{B}| \geq \rho^{2}+3 \rho$ and we are done. But, if both $S_{y}-e_{1}-e_{2}$ and $S_{y}-2 e_{1}-2 e_{2}$ are contained in $\mathcal{B}$, then $|(s+\mathcal{N}) \cap \mathcal{B}| \geq \rho^{2}+3 \rho-1$, $\left|\left(p_{1,1}+\mathcal{N}\right) \cap \mathcal{B}\right| \geq \rho^{2}+\rho-1$ and we are done.

Subcase 5.2.2. $\left|\psi^{-1}(s)\right|=2$.

Assume without loss of generality that $s=p_{1,-1}$ and that $\psi\left(p_{1,1}\right)=\psi\left(p_{-1,-1}\right)=$ $s$. Let $S_{y=\left(y_{1}, y_{2}\right)}$ be the square in $\left.\mathcal{B}\right|_{\mathcal{N}}$ adjacent to $p_{1,1}$ and $S_{z=\left(z_{1}, z_{2}\right)}$ be the square in $\left.\mathcal{B}\right|_{\mathcal{N}}$ adjacent to $p_{-1,-1}$. Since there are no corner pegs in $\bar{Q}_{1,1} \cup \bar{Q}_{-1,-1}$, we must have $\left.\mathcal{B}\right|_{\mathcal{N}} \subset\left[z, y+(\rho-1) e_{1}+(\rho-1) e_{2}\right]$. Since $\left.c(\mathcal{B}) \subset \mathcal{B}\right|_{\mathcal{N}}$ we must have $y_{2}-z_{2} \geq 2$ and $y_{1}-z_{1} \geq 2$.

Now, if there exists a corner peg $p$ in $\bar{Q}_{1,-1}$ such that $\mid\left\{\left.x \in \mathcal{B}\right|_{\mathcal{N}}:\|x-p\|_{\infty}=\right.$ $1\} \mid=5$, then $|(s+\mathcal{N}) \cap \mathcal{B}| \geq \rho^{2}+4 \rho$ and we are done. If

$$
\max \left\{\left|\left\{\left.x \in \mathcal{B}\right|_{\mathcal{N}}:\|x-p\|_{\infty}=1\right\}\right|: p \in \bar{Q}_{1,-1} \text { is a corner peg }\right\}=4,
$$

then $|(s+\mathcal{N}) \cap \mathcal{B}| \geq \rho^{2}+4 \rho-2,\left|\left(p_{1,1}+\mathcal{N}\right) \cap \mathcal{B}\right|,\left|\left(p_{-1,-1}+\mathcal{N}\right) \cap \mathcal{B}\right| \geq \rho^{2}+\rho-2$ and we are done. Finally, if $\max \left\{\left|\left\{\left.x \in \mathcal{B}\right|_{\mathcal{N}}:\|x-p\|_{\infty}=1\right\}\right|: p \in \bar{Q}_{1,-1}\right.$ is a corner peg $\}=$ 3 , then $|(s+\mathcal{N}) \cap \mathcal{B}| \geq \rho^{2}+3 \rho-1,\left|\left(p_{1,1}+\mathcal{N}\right) \cap \mathcal{B}\right|,\left|\left(p_{-1,-1}+\mathcal{N}\right) \cap \mathcal{B}\right| \geq \rho^{2}+\rho-1$ and we are done.

\section{Proof of the local Averaging Claim}

Here we employ a pairing strategy: we pair $x \in \varphi^{-1}(h)$ for which $\left|(x+\mathcal{N}) \cap A_{\infty}\right|>$ $2 \theta$ with $y \in \varphi^{-1}(h)$ such that $\left|(x+\mathcal{N}) \cap A_{\infty}\right|+\left|(y+\mathcal{N}) \cap A_{\infty}\right| \leq 4 \theta$. 
For ease of notation let $h$ be the origin (so $h+\mathcal{N}=\mathcal{N})$, and let $\omega^{\prime}=\lfloor\theta /(2 \rho+1)\rfloor$ (so $\omega=\max \left\{\omega^{\prime},\lfloor\rho / 2\rfloor+1\right\}$ ). First note that, since $h \notin A_{\infty},\left|\mathcal{N} \cap A_{\infty}\right|<\theta$. Since $|(x+\mathcal{N}) \backslash \mathcal{N}| \leq(2 \rho+1)\|x\|_{1}$ for all $x \in \mathbf{Z}^{2}$, it follows that

$$
\left|(x+\mathcal{N}) \cap A_{\infty}\right|<\theta+(2 \rho+1)\|x\|_{1} \text { for all } x \in \mathbf{Z}^{2} .
$$

So, for example, for those $x$ which satisfy $\|x\|_{1} \leq \omega^{\prime}$ we have $\left|(x+\mathcal{N}) \cap A_{\infty}\right|<2 \theta$.

Let $Y=\left\{x \in \varphi^{-1}(h):\|x\|_{1}>\omega^{\prime},\|x\|_{\infty} \leq \omega^{\prime}\right.$, and $\left.x \neq \pm \omega^{\prime} e_{1} \pm \omega^{\prime} e_{2}\right\}$. In order to define our pairings, we define a map $\mu: Y \rightarrow\left\{x \in \varphi^{-1}(h):\|x\|_{1}<\omega^{\prime}\right\}$. If $x=\left(\alpha x_{1}, \beta x_{2}\right)$ where $x_{1}, x_{2} \geq 0$ and $\alpha, \beta \in\{-1,1\}$, then

$$
\mu(x):=\left(\alpha\left(\omega^{\prime}-x_{2}\right), \beta\left(\omega^{\prime}-x_{1}\right)\right) .
$$

In words, $\mu$ is the reflection about the line $x_{1}+x_{2}=\omega^{\prime}$ in the first quadrant, the reflection about the line $-x_{1}+x_{2}=\omega^{\prime}$ in the second quadrant, and so on. Note that for all $x \in Y$

$$
\|x\|_{1}+\|\mu(x)\|_{1}=\omega^{\prime} .
$$

Claim 16. $x \in Y \Rightarrow \varphi(\mu(x))=h$.

Proof. Assume without loss of generality that $x=\left(x_{1}, x_{2}\right)$ with $0<x_{2} \leq x_{1}$. Let $h=h_{i}$ (i.e. $h$ is the $i^{\text {th }}$ hole in the ordering of $H$ ). From the definition of $\varphi$ we have

(a) $B_{\infty}\left(x, x_{1}-1\right) \subset A_{\infty}$,

(b) $B_{1}\left(x, x_{1}+x_{2}-1\right) \cap B_{\infty}\left(x, x_{1}\right) \subset A_{\infty}$,

(c) If $h_{j} \in B_{1}\left(x, x_{1}+x_{2}\right) \cap B_{\infty}\left(x, x_{1}\right)$, then $j \geq i$,

where $B_{n}(y, d)$ is the $\ell_{n}$ ball centered at $y$ with radius $d\left(B_{n}(y, d):=\left\{z \in \mathbf{Z}^{2}\right.\right.$ : $\left.\left.\|z-y\|_{n} \leq d\right\}\right)$. Note that $\|x-\mu(x)\|_{\infty}=x_{1}+x_{2}-\omega^{\prime}$ and $\|x-\mu(x)\|_{1}=$ $2\left(x_{1}+x_{2}-\omega^{\prime}\right)$. From the triangle inequality and $(a)$ we have $B_{\infty}\left(\mu(x), \omega^{\prime}-x_{2}-1\right) \subset$ $B_{\infty}\left(x, x_{1}-1\right) \subset A_{\infty}$; that is, $h$ is a closest hole to $\mu(x)$ in $\ell_{\infty}$ distance. From the triangle inequality and $(b)$ we have $B_{1}\left(\mu(x), 2 \omega^{\prime}-x_{2}-x_{1}-1\right) \cap B_{\infty}\left(\mu(x), \omega^{\prime}-x_{2}\right) \subset$ $B_{1}\left(x, x_{1}+x_{2}-1\right) \cap B_{\infty}\left(x, x_{1}\right) \subset A_{\infty}$; among those holes closest to $\mu(x)$ in $\ell_{\infty}$ distance $h$ is closest in $\ell_{1}$ distance. Finally, by the triangle inequality

$$
B_{1}\left(\mu(x), 2 \omega^{\prime}-x_{2}-x_{1}\right) \cap B_{\infty}\left(\mu(x), \omega^{\prime}-x_{2}\right) \subset B_{1}\left(x, x_{1}+x_{2}\right) \cap B_{\infty}\left(x, x_{1}\right) .
$$

So the hole with smallest index among the holes in $B_{1}\left(\mu(x), 2 \omega^{\prime}-x_{2}-x_{1}\right) \cap$ $B_{\infty}\left(\mu(x), \omega^{\prime}-x_{2}\right)$ is $h$, and $\varphi(\mu(x))=h$.

If $\mu$ is injective and $Y=\left\{x \in \varphi^{-1}(h):\|x\|_{1}>\omega^{\prime}\right\}$, then pairing $x$ with $\mu(x)$ works (note that we apply (28) and (29)):

$$
\begin{aligned}
\sum_{x \in \varphi^{-1}(h)}\left|(x+\mathcal{N}) \cap A_{\infty}\right|= & \sum_{x \in Y}\left|(x+\mathcal{N}) \cap A_{\infty}\right|+\left|(\mu(x)+\mathcal{N}) \cap A_{\infty}\right| \\
& +\sum_{x \in\left(\varphi^{-1}(h) \backslash Y \backslash \mu(Y)\right)}\left|(x+\mathcal{N}) \cap A_{\infty}\right| \\
< & \sum_{x \in Y}\left(\theta+(2 \rho+1)\|x\|_{1}+\theta+(2 \rho+1)\|\mu(x)\|_{1}\right) \\
& +\left|\varphi^{-1}(h) \backslash Y \backslash \mu(Y)\right| 2 \theta \\
= & |Y|\left(2 \theta+2 \omega^{\prime}(2 \rho+1)\right)+\left(\left|\varphi^{-1}(h)\right|-2|Y|\right) 2 \theta \\
\leq & |Y| 4 \theta+\left(\left|\varphi^{-1}(h)\right|-2|Y|\right) 2 \theta \\
= & 2 \theta\left|\varphi^{-1}(h)\right| .
\end{aligned}
$$


Unfortunately, $\mu$ is not always injective and $Y$ is not always $\left\{x \in \varphi^{-1}(h)\right.$ : $\left.\|x\|_{1}>\omega^{\prime}\right\}$. Elements of $\varphi^{-1}(h)$ on the coordinate axes may have two preimages under $\mu$, and we may have $Y \neq\left\{x \in \varphi^{-1}(h):\|x\|_{1}>\omega^{\prime}\right\}$ when $\omega^{\prime}<\omega$ (i.e. when $\rho^{2}<\theta \leq \rho^{2}+3 \rho / 2$ for odd $\rho$ and when $\rho^{2}<\theta \leq \rho^{2}+5 \rho / 2$ for even $\rho$ ). We now make two corrections to our pairing strategy to handle these two potential problems.

Correction 1. $\mu$ is not injective.

The potential conflicts in our pairing are within the sets

$$
L_{v}:=\left\{u \in B_{\infty}\left(\omega^{\prime}\right): u-2 \omega^{\prime} v \in B_{\infty}\left(\omega^{\prime}\right)\right\} \backslash\left\{ \pm \omega^{\prime} e_{1} \pm \omega^{\prime} e_{2}\right\}
$$

where $v \in\left\{ \pm e_{1}, \pm e_{2}\right\}$. We handle these conflicts by changing the definition of $\mu$ on $L_{v}$ when there exist $x, y \in L_{v} \cap \varphi^{-1}(h)$ with $\mu(x)=\mu(y)$. We show how this adjustment of $\mu$ is achieved when on $L:=L_{e_{2}}$; the adjustments for the other $L_{v}$ 's are the obvious symmetric analogs.

Let $L^{\prime}=\left\{\left(-a, \omega^{\prime}\right): 0<a<\omega^{\prime}\right.$ and $\left.\left(-a, \omega^{\prime}\right),\left(a, \omega^{\prime}\right) \in \varphi^{-1}(h)\right\}$; let $M=\{(0, b)$ : $\left.0<b<\omega^{\prime}\right\}$; and let $M^{\prime}=\left\{(0, b) \in M:\left(\omega^{\prime}-b, \omega^{\prime}\right),\left(b-\omega^{\prime}, \omega^{\prime}\right) \notin \varphi^{-1}(h)\right\}$.

Claim 17. If $L^{\prime} \neq \emptyset$, then $\varphi(M)=h$.

Proof. For some $a$ we have $B_{\infty}\left(\left(-a, \omega^{\prime}\right), \omega^{\prime}-1\right), B_{\infty}\left(\left(a, \omega^{\prime}\right), \omega^{\prime}-1\right) \subset A_{\infty}$. For an arbitrary $(0, b) \in M$ we have

$$
\begin{aligned}
B_{\infty}((0, b), b-1) & \subset B_{\infty}\left(\left(0, \omega^{\prime}\right), \omega^{\prime}-1\right) \\
& \subset B_{\infty}\left(\left(-a, \omega^{\prime}\right), \omega^{\prime}-1\right) \cup B_{\infty}\left(\left(a, \omega^{\prime}\right), \omega^{\prime}-1\right) \\
& \subset A_{\infty}
\end{aligned}
$$

and

$$
\begin{aligned}
B_{1}((0, b), b) \backslash A_{\infty} & \subset B_{1}((0, b), b) \backslash B_{\infty}\left(\left(0, \omega^{\prime}\right), \omega^{\prime}-1\right) \\
& =h .
\end{aligned}
$$

It follows that $\varphi(M)=h$.

Claim 18. If $\varphi\left(a, \omega^{\prime}\right)=\varphi\left(c, \omega^{\prime}\right)=h$ and $-\omega^{\prime}<a<b<c<\omega^{\prime}$, then $\varphi\left(b, \omega^{\prime}\right)=h$.

Proof. Since $B_{\infty}\left(\left(b, \omega^{\prime}\right), \omega^{\prime}-1\right) \subset B_{\infty}\left(\left(a, \omega^{\prime}\right), \omega^{\prime}-1\right) \cup B_{\infty}\left(\left(c, \omega^{\prime}\right), \omega^{\prime}-1\right), h$ is among the holes closest to $\left(b, \omega^{\prime}\right)$ in $\ell_{\infty}$ distance. Note that $\left\|\left(0,2 \omega^{\prime}\right)-\left(a, \omega^{\prime}\right)\right\|_{\infty}=\|(0,0)-$ $\left(a, \omega^{\prime}\right) \|_{\infty}$ and $\left\|\left(0,2 \omega^{\prime}\right)-\left(a, \omega^{\prime}\right)\right\|_{1}=\left\|(0,0)-\left(a, \omega^{\prime}\right)\right\|_{1}$. This implies that, since $\varphi\left(a, \omega^{\prime}\right)=(0,0)$, either $\left(0,2 \omega^{\prime}\right) \in A_{\infty}$ or $\left(0,2 \omega^{\prime}\right)$ follows $(0,0)$ in the ordering of $H$. In other words, the hole $(0,0)$ is chosen before $\left(0,2 \omega^{\prime}\right)$ in any tie-breaking situation. This observation finishes the proof because $B_{1}\left(\left(b, \omega^{\prime}\right), b+\omega^{\prime}-1\right) \cap B_{\infty}\left(\left(b, \omega^{\prime}\right), \omega^{\prime}\right) \subset$ $\left(B_{1}\left(\left(a, \omega^{\prime}\right), a+\omega^{\prime}-1\right) \cap B_{\infty}\left(\left(a, \omega^{\prime}\right), \omega^{\prime}\right)\right) \cup\left(B_{1}\left(\left(c, \omega^{\prime}\right), c+c \omega^{\prime}-1\right) \cap B_{\infty}\left(\left(c, \omega^{\prime}\right), \omega^{\prime}\right)\right) \subset$ $A_{\infty}$ and $B_{1}\left(\left(b, \omega^{\prime}\right), b+\omega^{\prime}\right) \cap B_{\infty}\left(\left(b, \omega^{\prime}\right), \omega^{\prime}\right) \backslash\left(B_{1}\left(\left(a, \omega^{\prime}\right), a+\omega^{\prime}-1\right) \cap B_{\infty}\left(\left(a, \omega^{\prime}\right), \omega^{\prime}\right)\right) \cup$ $\left(B_{1}\left(\left(c, \omega^{\prime}\right), c+c \omega^{\prime}-1\right) \cap B_{\infty}\left(\left(c, \omega^{\prime}\right), \omega^{\prime}\right)\right)=\left\{(0,0),\left(0,2 \omega^{\prime}\right)\right\}$.

It follows from Claim 18 that $M^{\prime}=\{(0, b): 0<b<m\}$ for some $m$.

Another important property of these sets is that $\left|M^{\prime}\right| \geq\left|L^{\prime}\right|$. This follows from $h \in \mathbf{Z}^{2} \backslash A_{\infty}$, is nontrivial, and is proven below. Assuming we have $\left|M^{\prime}\right| \geq\left|L^{\prime}\right|$ we change the definition of $\mu$ for the elements of $L^{\prime}$ in such a way that the restriction of $\mu$ to $L^{\prime}$ is a one-to-one map from $L^{\prime}$ to $M^{\prime}$. Since $M^{\prime}=\{(0, b): 0<b<m\}$, this new map will have the property that if $\mu\left(\left(-a, \omega^{\prime}\right)\right)=(0, b)$, then $b<\omega^{\prime}-a$ and therefore that $\left\|\left(-a, \omega^{\prime}\right)\right\|_{1}+\left\|\mu\left(-a, \omega^{\prime}\right)\right\|_{1} \leq 2 \omega^{\prime}$. This serves as a more than 
adequate replacement of (29) and it follows that the bounds given in (30) are valid for our new $\mu$.

It remains to show that $\left|M^{\prime}\right| \geq\left|L^{\prime}\right|$. This condition is equivalent to $\left|L \cap \varphi^{-1}(h)\right| \leq$ $\omega^{\prime}$. We show that $\left|L \cap \varphi^{-1}(h)\right| \leq \omega^{\prime}$ by contradiction; we show that $\left|L \cap \varphi^{-1}(h)\right| \geq$ $\omega^{\prime}+1$ contradicts $h \in \mathbf{Z}^{2} \backslash A_{\infty}$. We must divide this proof into two cases.

Correction 1.1. $\omega^{\prime} \geq\lfloor\rho / 2\rfloor+1$.

Here we are assuming $\omega^{\prime} \geq 3$. So the argument given here applies for $\rho \geq 4$. When $\rho=2,3$ very similar arguments can be used; they are left to the reader.

Assume $\left|L \cap \varphi^{-1}(h)\right| \geq \omega^{\prime}+1$. Claim 18 implies that $(1, \omega),(-1, \omega) \in \varphi^{-1}(h)$ and that (here we use $\omega^{\prime} \geq 3$ ) either $(2, \omega) \in \varphi^{-1}(h)$ or $(-2, \omega) \in \varphi^{-1}(h)$. Assume without loss of generality that $(2, \omega) \in \varphi^{-1}(h)$. Let $A=B_{\infty}\left(\left(-1, \omega^{\prime}\right), \omega^{\prime}-1\right) \cup$ $B_{\infty}\left(\left(1, \omega^{\prime}\right), \omega^{\prime}-1\right) \cup B_{\infty}\left(\left(2, \omega^{\prime}\right), \omega^{\prime}-1\right) \subset A_{\infty}$. If $\left(a, \omega^{\prime}\right) \in \varphi^{-1}(h) \backslash\left(0, \omega^{\prime}\right)$, then $B_{1}\left(\left(a, \omega^{\prime}\right),|a|+\omega^{\prime}-1\right) \cap B_{\infty}\left(\left(a, \omega^{\prime}\right), \omega^{\prime}\right) \subset A_{\infty}$ which implies that $(a, 0) \in A_{\infty}$. So, we know that both $A$ and $A^{\prime}:=\left\{(a, 0):\left(a, \omega^{\prime}\right) \in \varphi^{-1}(h) \backslash\left(0, \omega^{\prime}\right)\right\}$ are contained in $A_{\infty}$.

If $\omega^{\prime}=\rho$, then $\left|\mathcal{N} \cap A_{\infty}\right| \geq|\mathcal{N} \cap A|=2 \rho^{2}+\rho \geq \theta$, which is a contradiction. If $\omega^{\prime}<\rho$, then

$$
\begin{aligned}
\left|\mathcal{N} \cap A_{\infty}\right| & \geq|\mathcal{N} \cap A|+\left|\mathcal{N} \cap A^{\prime}\right| \\
& \geq \rho\left(2 \omega^{\prime}+2\right)+\left|A^{\prime}\right| \\
& \geq 2 \omega^{\prime} \rho+2 \rho+\omega^{\prime} \\
& \geq \theta .
\end{aligned}
$$

Correction 1.2. $\omega^{\prime}<\lfloor\rho / 2\rfloor+1$.

Assume $\left|L^{\prime \prime}:=L \cap \varphi^{-1}(h)\right|=\omega^{\prime}+1$. Consider the sets

$$
A^{\prime}:=\bigcup_{x \in L^{\prime \prime}} B_{\infty}\left(x, \omega^{\prime}-1\right), \quad A^{\prime \prime}:=\bigcup_{x \in L^{\prime \prime}} B_{1}\left(x,\|x\|_{1}-1\right) \cap B_{\infty}\left(x, \omega^{\prime}\right)
$$

and $A:=A^{\prime} \cup A^{\prime \prime}$. Since the furthest any element of $A$ is from the origin in $\ell_{\infty}$ distance is $2 \omega^{\prime}, A \subset \mathcal{N}$. Therefore, in order to get a contradiction we must simply show that $|A| \geq \theta$. Now, $\left|A^{\prime}\right|=\left(2 \omega^{\prime}-1\right)\left(2 \omega^{\prime}-1+\omega^{\prime}\right)$ and $\left|A^{\prime \prime}\right|=6\left(\omega^{\prime}-1\right)$ so $|A|=6\left(\omega^{\prime}\right)^{2}+\omega^{\prime}-5$.

There are three possible values for $\omega^{\prime}$ here. We finish this correction by showing $|A| \geq \theta$ in each of them. If $\rho$ is odd and $\omega^{\prime}=\rho / 2-1 / 2$, then $|A|=3 \rho^{2} / 2-5 \rho / 2-4$, and, since $\theta \leq \rho^{2}+3 / 2 \rho,|A| \geq \theta$ when $\rho \geq 9$. If $\rho$ is even and $\omega^{\prime}=\rho / 2-1$, then $|A|=3 \rho^{2} / 2-11 \rho / 2$, and, since $\theta \leq \rho^{2}+\rho / 2-1,|A| \geq \theta$ when $\rho \geq 12$. If $\rho$ is even and $\omega^{\prime}=\rho / 2$, then $|A|=3 \rho^{2} / 2+\rho / 2-5$, and, since $\theta \leq \rho^{2}+5 \rho / 2,|A| \geq \theta$ when $\rho \geq 6$.

We see that this argument fails for several small values of $\rho$. A different approach is needed to handle these cases. Rather than show $|\{x \in L: \varphi(x)=h\}| \leq$ $\omega^{\prime}$ we show that we cannot simultaneously have $\varphi(x)=h,\|x-h\|_{\infty}=\omega^{\prime}$ and $|x+\mathcal{N}| \cap A_{\infty}>2 \theta$. In other words, we handle these cases by showing that those elements of $\varphi^{-1}(h)$ for which our pairing breaks down do not need to be paired with anything. These calculations are left to the reader (note that Lemma 3 can be used here).

Correction 2. There exists $x$ such that $\varphi(x)=h$ and either $\|x-h\|_{\infty}>\omega^{\prime}$ or $x \in\left\{h \pm \omega^{\prime} e_{1} \pm \omega^{\prime} e_{2}\right\}$. 
We handle this problem with our pairing strategy by showing that in this situation $\left|(x+\mathcal{N}) \cap A_{\infty}\right|<2 \theta$; we show that those elements of $\varphi^{-1}(h)$ that are not contained in our pairings do not need to be paired. We argue indirectly. Assuming that $B_{\infty}\left(x, \omega^{\prime}\right) \backslash\left\{x \pm \omega^{\prime} e_{1} \pm \omega^{\prime} e_{2}\right\} \subset A_{\infty}$ and $\left|(x+\mathcal{N}) \cap A_{\infty}\right| \geq 2 \theta$, we will show that $B_{\infty}(x, \omega) \backslash\left\{x \pm \omega e_{1} \pm \omega e_{2}\right\} \subset A_{\infty}$. Since $h \in B_{\infty}(x, \omega) \backslash\left\{x \pm \omega e_{1} \pm \omega e_{2}\right\} \subset A_{\infty}$, this is a contradiction.

For ease of notation assume $x$ is the origin. Since we are assuming that $\mid(x+$ $\mathcal{N}) \cap A_{\infty} \mid \geq 2 \theta$ we can apply Lemma 3 :

$$
\text { if }\|z\|_{1} \leq \rho \text {, then either } z \in A_{\infty} \text { or }-z \in A_{\infty} .
$$

There are three ways in which we can have $\omega^{\prime}<\omega$. If $\rho$ is odd and $\omega^{\prime}<\omega$ we must have $\omega^{\prime}=\rho / 2-1 / 2$ and $\rho^{2}<\theta \leq \rho^{2}+3 \rho / 2-1 / 2$. If $\rho$ is even and $\omega^{\prime}<\omega$, then either $\omega^{\prime}=\rho / 2-1$ and $\rho^{2}<\theta \leq \rho^{2}+\rho / 2-1$ or $\omega^{\prime}=\rho / 2$ and $\rho^{2}+\rho / 2 \leq \theta \leq \rho^{2}+3 \rho / 2$. In each of these three cases we get $B_{\infty}(x, \omega) \backslash\left\{x \pm \omega e_{1} \pm \omega e_{2}\right\} \subset A_{\infty}$ in several steps beginning with $B_{\infty}\left(x, \omega^{\prime}\right) \backslash\left\{x \pm \omega^{\prime} e_{1} \pm \omega^{\prime} e_{2}\right\} \subset A_{\infty}$ and (31).

Note that in this situation (i.e. when $\theta$ is as above) $B_{\infty}\left(x, \omega^{\prime}\right) \backslash\left\{x \pm \omega^{\prime} e_{1} \pm \omega^{\prime} e_{2}\right\}$ actually generates the entire plane. So what we now prove can replace any mention of jagged blocks in the proof that the dynamics are omnivorous for these values of $\theta$.

Correction 2.1. $\rho$ is odd.

Let $A=B_{\infty}((\rho-1) / 2) \backslash\{( \pm((\rho-1) / 2), \pm((\rho-1) / 2))\}, C=\{( \pm((\rho-1) / 2)$, $\pm((\rho-1) / 2))\}, S_{1}=B_{\infty}((\rho+1) / 2) \cap B_{1}(\rho)$ and $S_{2}=B_{\infty}(\rho / 2+3 / 2) \cap B_{1}(\rho)$. By assumption $A \subset A_{\infty}$ and by (31) for $z \in C \cup S_{1} \cup S_{2}$ either $z$ or $-z$ is in $A_{\infty}$.

Step 1. $C \subset A_{\infty}$.

If $w \in C$, then $w+\mathcal{N}$ contains $A \cup C \cup S_{1}$. Thus,

$$
\begin{aligned}
\left|(w+\mathcal{N}) \cap A_{\infty}\right| & \geq|A|+|C| / 2+\left|S_{1}\right| / 2 \\
& =\rho^{2}-4+2+(4 \rho) / 2 \\
& =\rho^{2}+2 \rho-2 \\
& >\rho^{2}+3 \rho / 2 \\
& >\theta .
\end{aligned}
$$

Step 2. $S_{1} \backslash\left\{z:\|z\|_{1}=\rho\right\} \subset A_{\infty}$.

If $w \in S_{1} \backslash\left\{z:\|z\|_{1}=\rho\right\}$, then $w+\mathcal{N}$ contains $A \cup C$ as well as $3 / 4$ of both $S_{1}$ and $S_{2}$. For half of the $z \in S_{1} \cup S_{2}$ the set $w+\mathcal{N}$ contains both $z$ and $-z$. Thus,

$$
\begin{aligned}
\left|(w+\mathcal{N}) \cap A_{\infty}\right| & \geq|A|+|C|+\left|S_{1}\right| / 4+\left|S_{2}\right| / 4 \\
& =\rho^{2}-4+4+(4 \rho) / 4+(4(\rho-2)) / 4 \\
& =\rho^{2}+2 \rho-2 \\
& >\theta .
\end{aligned}
$$

Step 3. $S_{1} \cap\left\{z:\|z\|_{1}=\rho\right\} \subset A_{\infty}$.

If $w \in S_{1} \cap\left\{z:\|z\|_{1}=\rho\right\}$, then $w+\mathcal{N}$ contains $A \cup C$ as well as $3 / 4$ of the elements of $S_{1}$ that were shown to be in $A_{\infty}$ in Step 2. Thus,

$$
\begin{aligned}
\left|(w+\mathcal{N}) \cap A_{\infty}\right| & \geq|A|+|C|+3(\rho-2) \\
& =\rho^{2}+3 \rho-6 \\
& >\theta
\end{aligned}
$$


Correction 2.2. $\rho$ is even and $\omega^{\prime}=\rho / 2-1$.

Note that this case does not arise when $\rho=2$; we may assume $\rho \geq 4$. Let

$$
\begin{gathered}
A=B_{\infty}(\rho / 2-1) \backslash\{( \pm(\rho / 2-1), \pm(\rho / 2-1))\}, \\
S_{1}=B_{\infty}(\rho / 2) \backslash A, \\
S_{2}=B_{\infty}(\rho / 2+1) \backslash B_{\infty}(\rho / 2) \backslash\left\{z:\|z\|_{1}=\rho+2\right\},
\end{gathered}
$$

and

$$
S_{2}^{\prime}=S_{2} \backslash\left\{z:\|z\|_{1}=\rho+1\right\} .
$$

By assumption $A \subset A_{\infty}$ and by (31) for $z \in S_{1} \cup S_{2}^{\prime}$ either $z \in A_{\infty}$ or $-z \in A_{\infty}$.

Step 1. $S_{1} \backslash\left\{z:\|z\|_{1}=\rho\right\} \subset A_{\infty}$.

For $w \in S_{1} \backslash\left\{z:\|z\|_{1}=\rho\right\}$ the set $w+\mathcal{N}$ contains $A \cup S_{1}$ as well as $3 / 4$ of $S_{2}^{\prime}$. Therefore,

$$
\begin{aligned}
\left|(w+\mathcal{N}) \cap A_{\infty}\right| & \geq|A|+\left|S_{1}\right| / 2+\left|S_{2}^{\prime}\right| / 4 \\
& =(\rho-1)^{2}-4+(4 \rho+4) / 2+4(\rho-1) / 4 \\
& =\rho^{2}+\rho-2 \\
& \geq \rho^{2}+\rho / 2 \\
& >\theta .
\end{aligned}
$$

Step 2. $S_{1} \cap\left\{z:\|z\|_{1}=\rho\right\} \subset A_{\infty}$.

For $w \in S_{1} \cap\left\{z:\|z\|_{1}=\rho\right\}$ the set $w+\mathcal{N}$ contains $A \cup S_{1} \backslash\left\{z:\|z\|_{1}=\rho\right\}$. Thus, $\left|(w+\mathcal{N}) \cap A_{\infty}\right| \geq(\rho+1)^{2}-2 \geq \theta$.

Step 3. $S_{2} \subset A_{\infty}$.

For $w \in S_{2}$ the set $w+\mathcal{N}$ contains at least $\rho(\rho+1)$ elements of $S_{1} \cup A$. Since we know from Step 2 that $S_{1} \cup A \subset A_{\infty}$, we have $S_{2} \subset A_{\infty}$.

Correction 2.3. $\rho$ is even and $\omega^{\prime}=\rho / 2$.

In this argument we assume, for the sake of simplicity, that $\rho \geq 12$. The remaining cases are left to the reader (note that in some of these cases arguments slightly more involved than what we give here are necessary). Let $A=$ $B_{\infty}(\rho / 2) \backslash\{( \pm \rho / 2, \pm \rho / 2)\}, C=\{( \pm \rho / 2, \pm \rho / 2)\}, S_{1}=\left(B_{\infty}(\rho / 2+1) \backslash B_{\infty}(\rho / 2)\right) \cap$ $B_{1}(\rho+1), S_{1}^{\prime}=S_{1} \backslash\left\{z:\|z\|_{1}=\rho+1\right\}, S_{1}^{\prime \prime}=S_{1}^{\prime} \backslash\left\{z:\|z\|_{1}=\rho\right\}$, and $S_{2}=$ $B_{\infty}(\rho / 2+2) \cap B_{1}(\rho)$. By assumption $A \subset A_{\infty}$ and by (31) for $z \in C \cup S_{1}^{\prime} \cup S_{2}$ either $z$ or $-z$ is in $A_{\infty}$.

Step 1. $S_{1}^{\prime \prime} \subset A_{\infty}$.

If $w \in S_{1}^{\prime \prime}$, then $|(w+\mathcal{N}) \cap A|=\rho(\rho+1)-2$ and $w+\mathcal{N}$ contains $3 / 4$ of both $S_{1}^{\prime}$ and $S_{2}$. This gives

$$
\begin{aligned}
\left|(w+\mathcal{N}) \cap A_{\infty}\right| & \geq|(w+\mathcal{N}) \cap A|+\left|S_{1}^{\prime}\right| / 4+\left|S_{2}\right| / 4 \\
& =\rho(\rho+1)-2+4(\rho-1) / 4+4(\rho-3) / 4 \\
& =\rho^{2}+3 \rho-6 \\
& \geq \rho^{2}+5 \rho / 2 \\
& \geq \theta .
\end{aligned}
$$


Step 2. $C \subset A_{\infty}$

If $w \in C$, then $w+\mathcal{N}$ contains $A \cup C$ as well as $1 / 2$ of $S_{1}^{\prime \prime}$. Since $S_{1}^{\prime \prime} \subset A_{\infty}$ we have

$$
\begin{aligned}
\left|(w+\mathcal{N}) \cap A_{\infty}\right| & \geq|A|+|C| / 2+\left|S_{1}^{\prime \prime}\right| / 2 \\
& =(\rho+1)^{2}-4+2+2(\rho-3) \\
& =\rho^{2}+4 \rho-7 \\
& >\rho^{2}+5 \rho / 2 \\
& \geq \theta .
\end{aligned}
$$

Step 3. $S_{1} \subset A_{\infty}$.

If $w \in S_{1}$, then $|(w+\mathcal{N}) \cap(A \cup C)|=\rho(\rho+1)$ and $w+\mathcal{N}$ contains $1 / 2$ of $S_{1}^{\prime \prime}$. Thus

$$
\begin{aligned}
\left|(w+\mathcal{N}) \cap A_{\infty}\right| & \geq|(w+\mathcal{N}) \cap(A \cup C)|+\left|S_{1}^{\prime \prime}\right| / 2 \\
& =\rho(\rho+1)+4(\rho-3) / 2 \\
& =\rho^{2}+3 \rho-6 \\
& \geq \rho^{2}+5 \rho / 2 \\
& \geq \theta .
\end{aligned}
$$

\section{REFERENCES}

[C] E. Berlekamp, J. Conway, and R. Guy, Winning Ways for Your Mathematical Plays, Academic Press, New York, 1982. MR 84h:90091a

[ES] P. Erdős and J. Selfridge, On a combinatorial game, J. Comb. Theory A, 14(1973) 298-301. MR 48:5655

[GG1] J. Gravner and D. Griffeath, Threshold Growth Dynamics, Transactions of the AMS, 340(1993) 837-869. MR 94b:52006

[GG2] J. Gravner and D. Griffeath, First Passage Times for Discrete Threshold Growth Dynamics, Ann. Prob, to appear.

Department of Mathematics, Rutgers University, New Brunswick, New Jersey 08903 Current address: Department of Mathematics, 2-339, Massachusetts Institute of Technology, Cambridge, Massachusetts 02139

E-mail address: bohman@math.mit.edu 